\title{
Meso-Scale Fracture Simulation using an augmented Lagrangian Approach
}

International Journal of Damage Mechanics $\mathrm{XX}(\mathrm{X}): 1-28$

\begin{abstract}
A cohesive zone model implemented in an augmented Lagrangian functional is used for simulation of meso-scale fracture problems in this paper. The method originally developed by Lorentz is first presented in a rigorous variational framework. The equivalence between the stationary point of the one field problem and the saddle point of mixed formulation is proved by solving the double inequality of the mixed functional. An adaptation to simulate fracture phenomenon in the meso-scale via mesh modification is also presented as an algorithm to insert zero-thickness interface elements based on Lagrange multipliers, boarding the non-trivial task of the field interpolation for different crack paths (plain and tortuous). A suitable tool to study the matrix fracture and debonding phenomena in composites with strongly different component stiffnesses that avoids ill-conditioning matrices associated with intrinsic cohesive zone models is obtained. The method stability is discussed using a simple patch test. Some numerical applications to fracture problems taking into account the meso-structure and, particularly, the study of transverse failure of longitudinal fiber reinforced epoxy and the fracture in concrete specimens are included in the paper. Comparing the numerical results with the experimental results obtained by other researchers, the paper introduces a discussion about the influence of coarse-aggregate volume in meso-scale fracture mechanism in concrete L-shape specimens.
\end{abstract}

\section{Keywords}

Augmented Lagrangian Method, Cohesive Zone Model, Fracture Simulation, Extrinsic Traction-Separation Law, Composite Materials.

\section{INTRODUCTION}

Particle debonding and matrix cracking are usually involved in composite material behavior. Different numerical approaches have been proposed to deal with these problems where probably, the most popular is the concept of cohesive zone model (CZM) proposed by Dugdale (1960) and later by Barenblatt (1963). It was born as a generalization of the linear fracture mechanics theory, replacing the inter-locking and micro cracking zone by a transition stage called cohesive zone as shown in Figure 1. In this way, the singularity of the stress field on the crack tip is avoided. In the last two decades it has been increasingly studied in the computational mechanics community and it has been used to simulate a great number of static and dynamic problems.

In this context, the definition of a cohesive constitutive relationship, namely the traction-separation law (TSL) along the discontinuity surface, is a crucial requirement for the simulation of fracture behaviour. Several models have been proposed in the literature for this purpose and a recent review can be found in Park and Paulino (2011). The proposed models were used to analyse damage in different kinds of physical phenomenons, e.g. interface decohesion (Tvergaard and Hutchinson 1993), delamination (Geubelle and Baylor 1998; Pantano and Averill 2004; Turon et al. 2007; Nguyen and NguyenXuan 2013), debonding (Needleman 1987; Tvergaard 1990; Inglis et al. 2007), crack path in composites (Nguyen 2014; Wu et al. 2013; Cid Alfaro et al. 2010), etc, and have been implemented in different mathematical approaches like interface elements (Cerrone et al. 2014; Caggiano and Etse 2015), embedded-extended discontinuities (Unger et al. 2007; Linder et al. 2011), phase-field approaches (Verhoosel and de Borst 2013), mesh free methods (Rabczuk and Samaniego 2008) and isogeometric analysis (Corbett and Sauer 2015), among others. The use of interface elements has been widely extended due to its versatility to reproduce complex crack path, crack nucleation, crack branching and fragmentation, either in homogeneous or composites materials.

\footnotetext{
${ }^{1}$ Structure Institute, National University of Tucumán, San Miguel de Tucumán, Argentina.

${ }^{2}$ GIDMA, Department of Civil Engineering, National Technological University, Córdoba, Argentina.

${ }^{3}$ CONICET, Av. Rivadavia 1917, Buenos Aires, Argentina.
}

\section{Corresponding author:}

Nicolás A. Labanda, Av. Independencia 1800, San Miguel de Tucumán CP 4000, Argentina.

Email: nlabanda@facet.unt.edu.ar 
Interface elements can be categorized in two kinds: Intrinsic interface elements and extrinsic interface elements. The former was introduced for practical purposes in concrete fracture studies (Hillerborg et al. 1976), and it has the advantage of an easy mathematical and computational formulation with a straightforward parallelization and a low intrusive modification in classical finite element codes. As a counterpart, it leads to a spurious elastic regime in a 'pseudo un-cracked' solid and the risk of ill-conditioning of the tangent matrix due to the increase of the artificial compliance. The latter avoids numerical instabilities in the stiffness matrix in pre-failure regime but entails more convoluted formulations. Xu and Needleman (1993) proved that intrinsic TSL not only exhibits a non consistency due the initial slope but also has a strong mesh dependency.

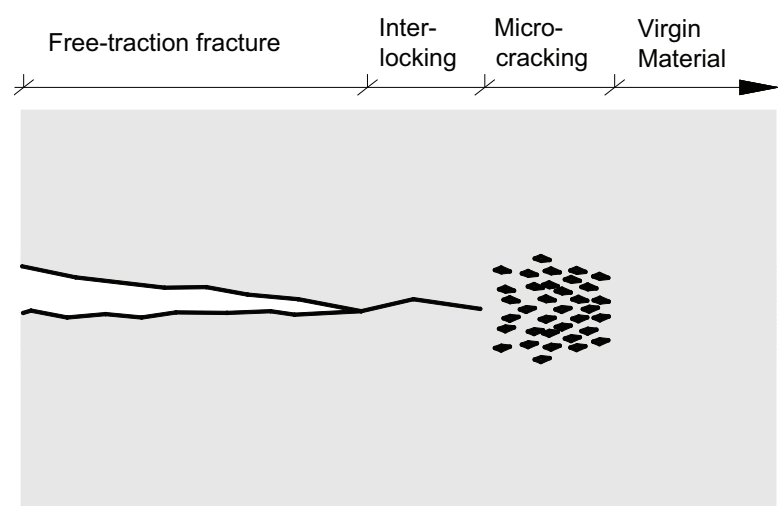

(a) Physical model for a fracture process

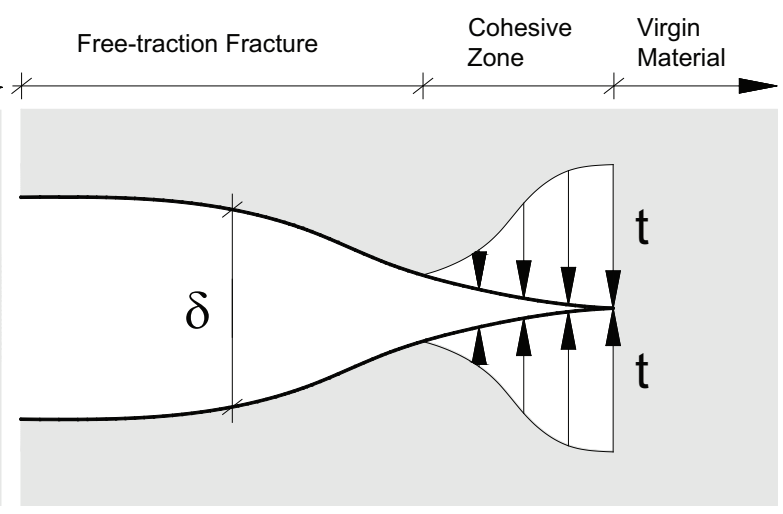

(b) Conceptual model for a fracture process considering a CZM.

Figure 1. Fracture modelling using the concept of CZM.

In order to avoid spurious load stages, several attempts to implement extrinsic TSL in different formulations have been carried out. Mergheim et al. (2004) presented a formulation based on an hybrid philosophy in which the equilibrium equation is splitted in two parts and they proposed a combination of the discontinuous Galerkin method to enforce the continuity of the continuum in the pre failure behaviour and a finite interface approach to control the post failure regime, using a switch parameter to jump from one to another. In a similar context, other researchers (Radovitzky et al. 2011; Nguyen 2014; Prechtel et al. 2011) used this kind of formulations to analyse more complex three dimensional fracture problems, crack propagation in fibre reinforced composites and dynamic problems. The discontinuous Galerkin method was also used to study the interface damage in fibre reinforced composites, e.g. the compact formulation developed by Truster and Masud (2013). The methodology presented there, was inspired in an augmented Lagrangian formulation of Lorentz (2008) and the coordination decomposition method presented in Fortin and Glowinski (1983), but with an enforcement of the TSL in collocation points driven by edge-projected stresses. Extrinsic TSL implemented using Lagrange multipliers have been less used for computational fracture mechanics. One of the first attempts can be traced back in Liu (1993) for the study of delamination and matrix cracking in cross-ply laminates. A decade later, other researchers like Elguedj et al. (2007) proposed an augmented Lagrangian formulation to model the fatigue phenomenon. It was developed in the context of the extended finite element method (X-FEM) and deals with unilateral contact in crack closure. Within the same finite element technology, Sadaba et al. (2015) developed a method for general cohesive interactions between crack faces, using a three field formulation (displacement, stresses and openings). Areias et al. (2004) proposed a formulation for finite displacement with embedded discontinuities, where a crack state parameter is fixed using a constraint term in the equilibrium equation. Lorentz (2008) presented the theoretical base of a unified augmented Lagrangian formulation for fracture mechanics, resulting in a method which is able to deal with unilateral contact and cohesive forces via a supplementary variable that enforces the jump displacement in so-called collocation points (Fortin and Glowinski 1983). An alternative approach has been published by Baiges et al. (2012), who proposed a method in the context of embedded discontinuities capable to weakly impose Dirichlet boundary conditions using an a-priori known parameter independent of the mesh and without adding extra degrees of freedom. Recently, Wheeler et al. (2014) developed a formulation based on phase field discontinuities, where an irreversibility constraint is fixed using an augmented Lagrangian penalization and an indicator function in the energy functional. They obtained a computational fracture model based on a functional minimization.

The combination of the concept of cohesive zone model and meso-mechanical simulations is promising to evaluate macroscopic responses of composites that are assumed to has three-phases at the meso-scale: matrix, particles (or aggregates) and the interphase or transition zone. Roelfstra et al. (1985) were the first using this approach for concrete and later, Bazant et al. (1990) proposed the random particle model. More recently, interface elements were introduced in meso-scale concrete models by Carol et al. (2001), López et al. (2008) and Du et al. (2013), among others. Cid Alfaro et al. (2010) used a classical interface technology and Nguyen (2014) presented a discontinuous Galerkin approach for transverse failure analysis of unidirectional fiber reinforced epoxy.

Although significant progress was made in this field, there is still a need of a mathematically well-defined mixed variational formulation and also, there is still a lack of rigorous and well-established numerical approaches which avoided so far the extensive use of the mixed interface element in computational fracture mechanics, specially for meso-scale applications. 
An approach based on the mixed formulation originally developed by Lorentz (2008) is applied to the meso-scale simulation of fracture problems in this paper. The mixed formulation is first written in a rigorous variational framework. The equivalence between the stationary point of the one field classic approach and the saddle point of the mixed formulation is shown by solving the double inequality in the continuum space. The traction separation law with linear unloading and all the details for its numerical implementation are presented. The finite element implementation in a Newton Raphson scheme is derived by solving a Taylor expansion of the discrete weak equilibrium problem. Additionally, a pre-process technique used to insert Lagrangian interface elements in a classical mesh is proposed and the detailed algorithm is presented. Finally some numerical examples, including debonding and matrix fracture are presented and compared with the results obtained by other researchers showing the capability of the method to obtain similar results with coarser meshes.

\section{VARIATIONAL FORMULATION}

The augmented Lagrangian method presented by Lorentz (2008) is described in this section in a rigorous variational framework and the saddle point problem is formally written starting from the direct formulation expressed as the summation of the body potential and a fracture potential. Then, the fracture potential is expressed via a supplementary variable subjected to a restriction using a augmented Lagrangian potential (Fortin and Glowinski 1983). Additionally, it is shown that the solution of the resulting saddle point problem corresponds to the solution of the stationary point of the direct formulation.

This mathematically rigorous proof represents a contribution of this paper that adds robustness to the method originally developed by Lorentz (2008).

\section{One field variational statement}

Let $\mathscr{L}^{\boldsymbol{u}}: \mathscr{U} \rightarrow \boldsymbol{R}$ be a system potential dependent on the body displacements $\boldsymbol{u}$ :

$$
\mathscr{L}^{\boldsymbol{u}}(\boldsymbol{u})=\mathscr{L}^{\mathcal{B}}(\boldsymbol{u})+\mathscr{L}^{\mathcal{F}}(\llbracket \boldsymbol{u} \rrbracket)
$$

with $\mathscr{L}^{\mathcal{B}}$ potential of a infinitesimally deformable volume $\Omega=\Omega^{-} \cup \Omega^{+}$and $\mathscr{L}^{\mathcal{F}}$ a fracture potential in $\Gamma$ defined as follows:

$$
\begin{aligned}
\mathscr{L}^{\mathcal{B}}(\boldsymbol{u}) & =\int_{\Omega \backslash \Gamma} \Phi(x, t) d \Omega-\int_{\Omega \backslash \Gamma} \boldsymbol{b}(x, t) \cdot \boldsymbol{u}(x, t) d \Omega-\eta(t) \int_{\partial^{t} \Omega} \overline{\boldsymbol{p}}(x) \cdot \boldsymbol{u}(x, t) d \partial^{t} \Omega, \\
\mathscr{L}^{\mathcal{F}}(\llbracket \boldsymbol{u} \rrbracket) & =\int_{\Gamma} \Psi(\llbracket \boldsymbol{u} \rrbracket, \kappa) d \Gamma,
\end{aligned}
$$

where $\partial^{t} \Omega$ is the Neumann boundary, with $\overline{\boldsymbol{p}}$ prescribed as an unitary load controlled by the parameter $\eta$ and $\partial^{u} \Omega$ the Dirichlet boundary presented in the Figure 2, and $\mathscr{U}(\Omega \backslash \Gamma)$ is the set of kinematically admissible displacement given by:

$$
\mathscr{U}(\Omega \backslash \Gamma)=\left\{\boldsymbol{u} \in \mathbf{H}^{1}(\Omega \backslash \Gamma) \wedge \llbracket \boldsymbol{u} \rrbracket \cdot \boldsymbol{n} \geq 0 \in \mathbf{H}^{\frac{1}{2}}(\Omega \backslash \Gamma):\left.\boldsymbol{u}\right|_{\partial^{u} \Omega}=\overline{\boldsymbol{u}}\right\}
$$

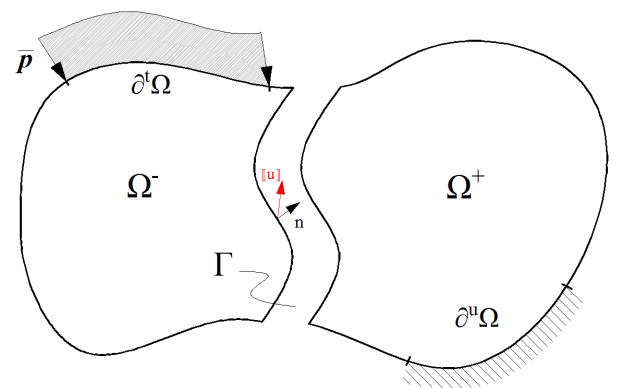

Figure 2. Domain decomposition of $\Omega$ considering a cohesive fracture $\Gamma$.

Furthermore, $\llbracket \bullet \rrbracket=\left.(\bullet)\right|_{\Gamma^{+}}-\left.(\bullet)\right|_{\Gamma^{-}}$represents the jump of the field $(\bullet)$ over the domain $\Gamma, \Phi$ is the elastic part of the Helmholtz free energy density, $\Psi$ is the energy density on the fracture $\Gamma, \eta \in \mathbb{R}$ is a load parameter defining the magnitude of the load that acts proportionally to a unit load $\bar{p} \in \mathbf{L}^{2}\left(\partial^{t} \Omega\right)$. The internal variable on the interface $\Gamma$ is denoted as $\kappa$ and $\boldsymbol{b} \in \mathbf{L}^{2}(\Omega \backslash \Gamma)$ is the volumetric force.

The elastic part of the Helmholtz free energy density is:

$$
\Phi=\frac{1}{2} \boldsymbol{\sigma}: \nabla^{s} \boldsymbol{u}
$$

where $\boldsymbol{\sigma}$ is the Cauchy tensor and $\nabla^{s} \boldsymbol{u}$ denotes the symmetric part of the displacement field gradient. 
Following Lorentz (2008) the cohesive energy $\Psi$ is defined including an indicator function $\boldsymbol{I}_{\mathbb{R}^{+}}$is included to avoid penetration between crack lips:

$$
\boldsymbol{I}_{\mathbb{R}^{+}}(\llbracket \boldsymbol{u} \rrbracket)=\left\{\begin{array}{ccc}
0 & \text { if } & \llbracket \boldsymbol{u} \rrbracket \cdot \boldsymbol{n} \geq 0 \\
+\infty & \text { if } & \llbracket \boldsymbol{u} \rrbracket \cdot \boldsymbol{n}<0
\end{array},\right.
$$

being $\boldsymbol{n}$ crack normal vector. The pseudo-potential over the crack $\Gamma$ is:

$$
\Psi(\llbracket \boldsymbol{u} \rrbracket, \kappa)=\boldsymbol{I}_{\mathbb{R}^{+}}\left(\llbracket \boldsymbol{u} \rrbracket_{\boldsymbol{n}}\right)+\psi(\llbracket \boldsymbol{u} \rrbracket, \kappa),
$$

with $\psi: \mathbb{R}^{+} \rightarrow \mathbb{R}$ representing the cohesive potential function.

The equilibrium problem can be stated as follows:

$$
\min _{\boldsymbol{u} \in \mathscr{U}} \mathscr{L}^{\boldsymbol{u}}(\boldsymbol{u})
$$

\section{Mixed variational statement}

The functional $\mathscr{L}^{\boldsymbol{u}}$ is reformulated using an augmented Lagrangian method (Lorentz 2008) and later, the decomposition coordination method (or collocation points method) proposed by Fortin and Glowinski (1983). In this way, a decoupling of the formulation into a global linear problem, and a family of local problems solved in the collocation points is carried out, where these two sets of problems are coordinated by Lagrange multipliers. Then, the direct solution of the problem (7) is replaced by a classical saddle point problem.

A supplementary variable $\boldsymbol{\delta}$ subjected to the restriction $\llbracket \boldsymbol{u} \rrbracket-\boldsymbol{\delta}=0$ is introduced, and the equilibrium problem stated in equation (7) can be rewritten as:

$$
\left\{\begin{array}{l}
\min _{(\boldsymbol{u}, \boldsymbol{\delta}) \in \mathscr{V} \times \mathscr{W}} \mathscr{L}^{\mathcal{B}}(\boldsymbol{u})+\mathscr{L}^{\mathcal{F}}(\boldsymbol{\delta}) \\
\text { subject to } \llbracket \boldsymbol{u} \rrbracket-\boldsymbol{\delta}=0
\end{array}\right.
$$

Associated to problem (8) an augmented Lagrangian functional $\mathscr{L}: \mathscr{V} \times \mathscr{W} \times \mathscr{X} \rightarrow \boldsymbol{R}$ can be defined as follows:

$$
\mathscr{L}(\boldsymbol{u} ; \boldsymbol{\delta} ; \boldsymbol{\lambda})=\mathscr{L}_{\gamma}^{\mathcal{B}}(\boldsymbol{u} ; \boldsymbol{\delta})+\mathscr{L}^{\mathcal{F}}(\boldsymbol{\delta})+\mathscr{L}^{\mathcal{C}}(\boldsymbol{u} ; \boldsymbol{\delta} ; \boldsymbol{\lambda})
$$

where:

$$
\begin{aligned}
& \mathscr{L}_{\gamma}^{\mathcal{B}}(\boldsymbol{u}, \boldsymbol{\delta})=\mathscr{L}^{\mathcal{B}}(\boldsymbol{u})+\int_{\Gamma} \frac{\gamma}{2}(\llbracket \boldsymbol{u} \rrbracket-\boldsymbol{\delta})^{2} d \Gamma, \\
& \mathscr{L}^{\mathcal{C}}(\boldsymbol{u} ; \boldsymbol{\delta}, \boldsymbol{\lambda})=\int_{\Gamma} \boldsymbol{\lambda} \cdot(\llbracket \boldsymbol{u} \rrbracket-\boldsymbol{\delta}) d \Gamma,
\end{aligned}
$$

where $\boldsymbol{\lambda}$ is the Lagrange multipliers field and $\gamma$ is the penalty parameter. Note that the second term in equation (9), corresponding to the cohesive energy density $\Psi$, becomes a function of the supplementary variable. This variable is controlled in the third term by the Lagrange multipliers $\boldsymbol{\lambda}$ fixing in a weak sense the restriction $\llbracket \boldsymbol{u} \rrbracket-\boldsymbol{\delta}=0$. The mechanical equilibrium problem leads to a saddle point problem and, in contrast with the original approach (Lorentz 2008), it is rewritten as the following double inequality problem as follows: Find $(\boldsymbol{u}, \boldsymbol{\delta}, \boldsymbol{\lambda}) \in \mathscr{V} \times \mathscr{W} \times \mathscr{X}$ s.t.

$$
\mathscr{L}(\boldsymbol{u}, \boldsymbol{\delta}, \boldsymbol{\mu}) \leq \mathscr{L}(\boldsymbol{u}, \boldsymbol{\delta}, \boldsymbol{\lambda}) \leq \mathscr{L}(\boldsymbol{v}, \boldsymbol{p}, \boldsymbol{\lambda}), \forall(\boldsymbol{v}, \boldsymbol{p}, \boldsymbol{\mu}) \in \mathscr{V} \times \mathscr{W} \times \mathscr{X}
$$

It will be shown that the solution of the double inequality (11) presented above is equivalent to the solution of the following stationary point problem:

$$
\underbrace{\partial_{\boldsymbol{u}} \mathscr{L} \cdot \delta \boldsymbol{u}}_{\mathscr{G}_{1}}+\underbrace{\partial_{\boldsymbol{\lambda}} \mathscr{L} \cdot \delta \boldsymbol{\lambda}}_{\mathscr{G}_{2}}+\underbrace{\partial_{\boldsymbol{\delta}} \mathscr{L} \cdot \delta \boldsymbol{\delta}}_{\mathscr{G}_{0}}=0, \quad \forall(\delta \boldsymbol{u}, \delta \boldsymbol{\delta}, \delta \boldsymbol{\lambda}) \in \mathscr{V} \times \mathscr{W} \times \mathscr{X} .
$$

Being $\mathscr{V}$ the space of kinematically admissible displacements variations $\delta \boldsymbol{u}, \mathscr{X}$ the space of kinematically admissible Lagrange multipliers variations $\delta \boldsymbol{\lambda}$ and in the same way, $\mathscr{W}$ the space of kinematically admissible supplementary variable variations $\delta \delta$, given by:

$$
\begin{aligned}
& \mathscr{V}(\Omega \backslash \Gamma)=\left\{\boldsymbol{v} \in \mathbf{H}^{1} \wedge \llbracket \boldsymbol{v} \rrbracket \in \mathbf{H}^{\frac{1}{2}}:\left.\boldsymbol{v}\right|_{\partial \boldsymbol{u}_{\Omega}}=0\right\}, \\
& \mathscr{W}(\Omega \backslash \Gamma)=\left\{\boldsymbol{w} \mid \boldsymbol{w} \in \mathbf{H}_{00}^{\frac{1}{2}}: \boldsymbol{w} \cdot \boldsymbol{n} \geq 0, \forall \boldsymbol{w} \in \Gamma\right\}, \\
& \mathscr{X}(\Omega \backslash \Gamma)=\left\{\boldsymbol{x} \mid \boldsymbol{x} \in \mathbf{H}^{-\frac{1}{2}}\right\},
\end{aligned}
$$


where the space $\mathbf{H}_{00}^{\frac{1}{2}}$ is a subspace of $\mathbf{H}^{\frac{1}{2}}$ introduced by Lions (1972). The operator $\partial_{\circ}(\cdot)$ represents the sub gradient or generalized derivative of the functional $(\cdot)$ respect to $\circ$. The sub gradient is equal to the partial derivative $\frac{\partial(\cdot)}{\partial \circ}$ only when $(\cdot)$ is differentiable, see Clarke (1978). The equilibrium equations of the fracture problem can be obtained from each term of equation (12) as:

$$
\begin{array}{rlrl}
\mathscr{G}_{0}(\boldsymbol{u} ; \boldsymbol{\delta} ; \boldsymbol{\lambda}) & =\int_{\Gamma}\left[\partial_{\boldsymbol{\delta}} \Psi(\boldsymbol{\delta}, \kappa)-\boldsymbol{\lambda}-\gamma(\llbracket \boldsymbol{u} \rrbracket-\boldsymbol{\delta})\right] \cdot \delta \boldsymbol{\delta} d \Gamma, & & \\
\mathscr{G}_{1}(\boldsymbol{u} ; \boldsymbol{\delta} ; \boldsymbol{\lambda}) & =\int_{\Omega \backslash \Gamma} \boldsymbol{\sigma}(x, t): \nabla^{s} \delta \boldsymbol{u}(x, t) d \Omega+\int_{\Gamma}[\boldsymbol{\lambda}+\gamma(\llbracket \boldsymbol{W} \rrbracket-\boldsymbol{\delta})] \cdot \llbracket \delta \boldsymbol{u} \rrbracket d \Gamma- & \\
& -\eta(t) \int_{\partial^{t} \Omega} \overline{\boldsymbol{p}}(x) \cdot \delta \boldsymbol{u} d \partial^{t} \Omega-\int_{\Omega \backslash \Gamma} \boldsymbol{b}(x, t) \cdot \delta \boldsymbol{u} d \Omega, & \forall \delta \boldsymbol{u} \in \mathscr{V}, \\
\mathscr{G}_{2}(\boldsymbol{u} ; \boldsymbol{\delta} ; \boldsymbol{\lambda}) & =\int_{\Gamma}(\llbracket \boldsymbol{u} \rrbracket-\boldsymbol{\delta}) \cdot \delta \boldsymbol{\lambda} d \Gamma & & \forall \delta \boldsymbol{\lambda} \in \mathscr{X} .
\end{array}
$$

The operator $\mathscr{G}_{0}$ represents a non differentiable equation which relates the constitutive behaviour of the variable $t \in \partial_{\boldsymbol{\delta}} \Psi$ with the Lagrange multipliers, from which a generalization of Hertz-Signorini-Moreau condition for contact problems is obtained (Yastrebov 2013). The discretized supplementary variable can be obtained from this functional as a function of the displacements and Lagrange multipliers $\boldsymbol{\delta}_{h}=\boldsymbol{\delta}(\boldsymbol{u} ; \boldsymbol{\lambda})$, using the coordination decomposition method. Then, the problem is reduced to: For some $\gamma \in \mathbb{R}^{+}$and $\eta \in \mathbb{R}$, find $(\boldsymbol{u} ; \boldsymbol{\lambda}) \in \mathscr{V} \times \mathscr{X}$ such that

$$
\begin{aligned}
& \mathscr{G}_{1}(\boldsymbol{u} ; \boldsymbol{\lambda})=0, \quad \forall \delta \boldsymbol{u} \in \mathscr{V}, \\
& \mathscr{G}_{2}(\boldsymbol{u} ; \boldsymbol{\lambda})=0, \quad \forall \delta \boldsymbol{\lambda} \in \mathscr{X},
\end{aligned}
$$

where the operators $\mathscr{G}_{1}$ and $\mathscr{G}_{2}$ were properly introduced in (14); and with $\boldsymbol{\delta}_{h}(\boldsymbol{u} ; \boldsymbol{\lambda}) \in \operatorname{ker} \mathscr{G}_{0}(\boldsymbol{u} ; \boldsymbol{\delta} ; \boldsymbol{\lambda}) \subset \mathscr{W}_{h}$ where $\boldsymbol{u}$ and $\lambda$ are fixed in collocation points.

\section{Equivalence between one-field problem solution and the mixed-formulation problem solution}

The equivalence between the solution of the direct minimization problem (7) and the saddle point problem (11) is analyzed in this section showing also the equivalence of the saddle point (Equation (11)), with the stationary point of the augmented Lagrangian functional (Equation (12)).

Let us define the space of kinematically admissible displacements $\mathscr{K}$ :

$$
\mathscr{K}=\left\{\boldsymbol{v} \in \mathbf{H}^{1}:(J \boldsymbol{v}) \cdot \boldsymbol{n} \geq 0 \text { in } \Gamma \wedge \boldsymbol{v}=0 \text { in } \partial^{\boldsymbol{u}} \Omega\right\} \subseteq \mathscr{V} \subseteq \mathscr{U} .
$$

The operator $J \in \mathcal{L}(\mathscr{V}, \mathscr{W})$ defined by $J: \boldsymbol{v} \in \mathscr{V} \mapsto \llbracket \boldsymbol{v} \rrbracket=J \boldsymbol{v} \in \mathscr{W}$, maps bulk displacements in gaps onto the fracture. In the following, the apostrophe is used to indicate the dual space.

Proposition 1. Let $\mathscr{L}_{\mathcal{B}}$ and $\mathscr{L}_{\mathcal{F}}$ (equation (2)) be continuously differentiable functionals in $\mathscr{V}(\Omega \backslash \Gamma)$ and $\exists k>0$ : $\forall \boldsymbol{u}, \boldsymbol{v} \in \mathscr{V},\left\|\partial \mathscr{L}^{\mathcal{F}}(\boldsymbol{u})-\partial \mathscr{L}^{\mathcal{F}}(\boldsymbol{v})\right\|_{\mathscr{W}^{\prime}} \leq k\|\boldsymbol{u}-\boldsymbol{v}\|_{\mathscr{W}}$, then the functional in Equation (1) is differentiable such that a solution $\boldsymbol{u}$ of the problem (7) satisfies:

$$
\int_{\Omega \backslash \Gamma} \partial \mathscr{L}^{\mathcal{B}} \cdot(\boldsymbol{v}-\boldsymbol{u}) d \Omega+\int_{\Gamma} \partial \mathscr{L}^{\mathcal{F}} \cdot \llbracket \boldsymbol{v}-\boldsymbol{u} \rrbracket d \Gamma \geq 0, \quad \forall \boldsymbol{v} \in \mathscr{K} .
$$

Furthermore, if $\mathscr{L}^{\mathcal{B}}$ is $\mathscr{V}$-elliptic with constant $\theta_{\mathcal{B}}$ and considering a continuity constant $c_{J}$ for $J$ operator, it can be shown that $\exists \alpha>0 \in \mathbb{R}$ such that $\mathscr{L}^{u}$ becomes strongly monotone, so the problem (7) has unique solution.

Remark 1. Doyen et al. (2010) ) probed that the constant is $\alpha=\theta^{\mathcal{B}}-k c_{J} \boldsymbol{c}_{\mathscr{X}}$, and it can be related with physical properties of the numerical model, being $\theta^{\mathcal{B}}$ proportional to the norm of the constitutive tensor in the bulk phase $\Omega$. The Lipschitz continuity constant $k$ grows with the decrease of cohesive forces. The continuity constant $\boldsymbol{c}_{J} \boldsymbol{c}_{\mathscr{X}}$ tends to zero when the dimensional measure $|\Gamma|$ tends to zero.

In the following proposition, the results reported by Chen (2001) is extended and adapted to cohesive problems relating the Lagrange multipliers with the fracture potential. In this way, this paper contributes to endowing the Lagrange multipliers with a physical meaning.

Proposition 2. Let $\boldsymbol{u} \in \mathscr{K}$ be the unique solution of problem (7) (proposition 1), then the augmented Lagrangian defined in Equation (9) has an unique saddle point $(\boldsymbol{u}, \boldsymbol{\delta}, \boldsymbol{\lambda}) \in \mathscr{V} \times \mathscr{W} \times \mathscr{X}$ such that:

$$
\mathscr{L}(\boldsymbol{u}, \boldsymbol{\delta}, \boldsymbol{\mu}) \leq \mathscr{L}(\boldsymbol{u}, \boldsymbol{\delta}, \boldsymbol{\lambda}) \leq \mathscr{L}(\boldsymbol{v}, \boldsymbol{p}, \boldsymbol{\lambda}), \forall(\boldsymbol{v}, \boldsymbol{p}, \boldsymbol{\mu}) \in \mathscr{V} \times \mathscr{W} \times \mathscr{X}
$$

Moreover, the following relations can be proved: 


$$
\boldsymbol{\lambda}=-\boldsymbol{\sigma} \cdot \boldsymbol{n}, \boldsymbol{\delta} \cdot \boldsymbol{n}=(J \boldsymbol{u}) \cdot \boldsymbol{n} \geq 0 \text { and } \boldsymbol{\lambda}=\partial_{\boldsymbol{\delta}} \mathscr{L}^{\mathcal{F}},
$$

when the derivative is defined (active cohesion).

Proof. $(\Leftarrow$ Suppose that $(\boldsymbol{u}, \boldsymbol{\delta}, \boldsymbol{\lambda}) \in \mathscr{V} \times \mathscr{W} \times \mathscr{X}$ is a saddle point of $\mathscr{L}$, then $\boldsymbol{u} \in \mathscr{K} \subseteq \mathscr{V}$ is a minimum of functional defined in Equation (1) and consequently equations (19) holds ).

Taking the left inequality of (18):

$$
\begin{gathered}
\mathscr{L}(\boldsymbol{u}, \boldsymbol{\delta}, \boldsymbol{\mu}) \leq \mathscr{L}(\boldsymbol{u}, \boldsymbol{\delta}, \boldsymbol{\lambda}), \forall \boldsymbol{\mu} \in \mathscr{X} \\
\int_{\Gamma} \boldsymbol{\mu} \cdot(J \boldsymbol{u}-\boldsymbol{\delta}) d \Gamma \leq \int_{\Gamma} \boldsymbol{\lambda} \cdot(J \boldsymbol{u}-\boldsymbol{\delta}) d \Gamma, \forall \boldsymbol{\mu} \in \mathscr{X} .
\end{gathered}
$$

As $\mathscr{X}$ is a linear space it follows that, by taking $\boldsymbol{\mu}=\boldsymbol{\lambda}+\boldsymbol{\pi} \in \mathscr{X}, \forall \boldsymbol{\pi} \in \mathscr{X}$, the last equation leads to

$$
\int_{\Gamma} \boldsymbol{\pi} \cdot(J \boldsymbol{u}-\boldsymbol{\delta}) d \Gamma \leq 0, \forall \boldsymbol{\pi} \in \mathscr{X}
$$

but if $\boldsymbol{\pi} \in \mathscr{X}$, by linearity $-\boldsymbol{\pi} \in \mathscr{X}$, resulting in $\int_{\Gamma} \boldsymbol{\pi} \cdot(J \boldsymbol{u}-\boldsymbol{\delta}) d \Gamma=0, \forall \boldsymbol{\pi} \in \mathscr{X}$. The only way to vanish the integral is fulfilling $J \boldsymbol{u}=\boldsymbol{\delta}$ over the fracture domain $\Gamma$. By taking the right inequality:

$$
\begin{aligned}
\mathscr{L}(\boldsymbol{u}, \boldsymbol{\delta}, \boldsymbol{\lambda}) \leq \mathscr{L}(\boldsymbol{v}, \boldsymbol{p}, \boldsymbol{\lambda}), \quad \forall(\boldsymbol{v}, \boldsymbol{p}) \in \mathscr{V} \times \mathscr{W}, \\
\mathscr{L}^{\mathcal{B}}(\boldsymbol{u})+\mathscr{L}^{\mathcal{F}}(\boldsymbol{\delta})+\int_{\Gamma} \boldsymbol{\lambda} \cdot \underbrace{(\llbracket \boldsymbol{u} \rrbracket-\boldsymbol{\delta})}_{=0} d \Gamma+\int_{\Gamma} \frac{\gamma}{2} \underbrace{(\llbracket \boldsymbol{u} \rrbracket-\boldsymbol{\delta})^{2}}_{=0} d \Gamma \leq \mathscr{L}^{\mathcal{B}}(\boldsymbol{v})+\mathscr{L}^{\mathcal{F}}(\boldsymbol{p})+ \\
+\int_{\Gamma} \boldsymbol{\lambda} \cdot(\llbracket \boldsymbol{v} \rrbracket-\boldsymbol{p}) d \Gamma+\int_{\Gamma} \frac{\gamma}{2}(\llbracket \boldsymbol{v} \rrbracket-\boldsymbol{p})^{2} d \Gamma .
\end{aligned}
$$

Due to the indicator function in $\mathscr{L}^{\mathcal{F}}(\boldsymbol{\delta})$, the restriction $\boldsymbol{\delta} \cdot \boldsymbol{n} \geq 0$ should be added, otherwise the inequality does not hold. If $\boldsymbol{v} \in \mathscr{K}$, and taking $\boldsymbol{p}=J \boldsymbol{v}$ over $\Gamma$ :

$$
\mathscr{L}^{\mathcal{B}}(\boldsymbol{u})+\mathscr{L}^{\mathcal{F}}(J \boldsymbol{u}) \leq \mathscr{L}^{\mathcal{B}}(\boldsymbol{v})+\mathscr{L}^{\mathcal{F}}(J \boldsymbol{v})+\int_{\Gamma} \boldsymbol{\lambda} \cdot \underbrace{(\llbracket \boldsymbol{v} \rrbracket-\boldsymbol{p})}_{=0} d \Gamma+\int_{\Gamma} \frac{\gamma}{2} \underbrace{(\llbracket \boldsymbol{v} \rrbracket-\boldsymbol{p})^{2}}_{=0} d \Gamma .
$$

Finally

$$
\mathscr{L}^{\boldsymbol{u}}(\boldsymbol{u}) \leq \mathscr{L}^{\boldsymbol{u}}(\boldsymbol{v}), \quad \forall \boldsymbol{v} \in \mathscr{K}
$$

being $\boldsymbol{u} \in \mathscr{K}$ the minimum of $\mathscr{L}^{\boldsymbol{u}}$ and solution of the problem (7).

Let us prove the first postulate in equation (19) by starting with equation (21). Now, taking $\boldsymbol{p}=\boldsymbol{\delta}$ and a vector $\boldsymbol{v}=\boldsymbol{u}+t(\boldsymbol{w}-\boldsymbol{u}) \in \mathscr{V}$ with $t \in[0,1]$, the following relations has been obtained:

$$
\begin{aligned}
& \mathscr{L}^{\mathcal{B}}(\boldsymbol{u}+t(\boldsymbol{w}-\boldsymbol{u}))-\mathscr{L}^{\mathcal{B}}(\boldsymbol{u})+\int_{\Gamma} \boldsymbol{\lambda} \cdot(t(\llbracket \boldsymbol{w} \rrbracket-\llbracket \boldsymbol{u} \rrbracket)) d \Gamma+\int_{\Gamma} \frac{\gamma}{2}(t(\llbracket \boldsymbol{w} \rrbracket-\llbracket \boldsymbol{u} \rrbracket))^{2} d \Gamma \geq 0, \\
& \frac{\mathscr{L}^{\mathcal{B}}(\boldsymbol{u}+t(\boldsymbol{w}-\boldsymbol{u}))-\mathscr{L}^{\mathcal{B}}(\boldsymbol{u})}{t}+\int_{\Gamma} \boldsymbol{\lambda} \cdot(\llbracket \boldsymbol{w} \rrbracket-\llbracket \boldsymbol{u} \rrbracket) d \Gamma+\int_{\Gamma} t \frac{\gamma}{2}((\llbracket \boldsymbol{w} \rrbracket-\llbracket \boldsymbol{u} \rrbracket))^{2} d \Gamma \geq 0 .
\end{aligned}
$$

Taking the limit with $t \rightarrow 0^{+}$in both sides

$$
\begin{aligned}
\int_{\Omega \backslash \Gamma} \boldsymbol{\sigma}(x, t): \nabla^{s}(\boldsymbol{w}-\boldsymbol{u}) d \Omega+\int_{\Gamma} \boldsymbol{\lambda} \cdot J(\boldsymbol{w}-\boldsymbol{u}) d \Gamma & \geq \eta(t) \int_{\partial^{t} \Omega} \overline{\boldsymbol{p}}(x) \cdot(\boldsymbol{w}-\boldsymbol{u}) d \partial^{t} \Omega+ \\
& +\int_{\Omega \backslash \Gamma} \boldsymbol{b}(x, t) \cdot(\boldsymbol{w}-\boldsymbol{u}) d \Omega
\end{aligned}
$$

By making $\boldsymbol{v}=(\boldsymbol{w}-\boldsymbol{u}) \in \mathscr{V}$, it can be proved that:

$$
\int_{\Omega \backslash \Gamma} \boldsymbol{\sigma}(x, t): \nabla^{s} \boldsymbol{v} d \Omega+\int_{\Gamma} \boldsymbol{\lambda} \cdot J \boldsymbol{v} d \Gamma=\eta(t) \int_{\partial^{t} \Omega} \overline{\boldsymbol{p}}(x) \cdot \boldsymbol{v} d \partial^{t} \Omega+\int_{\Omega \backslash \Gamma} \boldsymbol{b}(x, t) \cdot \boldsymbol{v} d \Omega .
$$


Applying Green's theorem, the first term of the first member can be written as:

$$
\int_{\Omega \backslash \Gamma} \boldsymbol{\sigma}(x, t): \nabla^{s} \boldsymbol{v} d \Omega=-\int_{\Omega \backslash \Gamma}(\operatorname{div} \sigma) \cdot \boldsymbol{v} d \Omega+\int_{\partial^{t} \Omega}(\boldsymbol{\sigma} \cdot \boldsymbol{n}) \cdot \boldsymbol{v} d \partial^{t} \Omega+\int_{\Gamma}(\boldsymbol{\sigma} \cdot \boldsymbol{n}) J \boldsymbol{v} d \Gamma .
$$

Substituting equation (23) in equation (22), the relation $\boldsymbol{\lambda}=-\boldsymbol{\sigma} \cdot \boldsymbol{n}$ is obtained.

To prove the last relation in (19), lets take a vector $\boldsymbol{p}=\boldsymbol{\delta}+t(\boldsymbol{\vartheta}-\boldsymbol{\delta})$ with $t \in[0,1]$ and $\boldsymbol{u}=\boldsymbol{v}$ in Eq. (21) to get the relation:

$$
\int_{\Gamma}\left(\partial_{\boldsymbol{\delta}} \mathscr{L}^{\mathcal{F}}-\boldsymbol{\lambda}\right) \cdot(\boldsymbol{\vartheta}-\boldsymbol{\delta}) d \Gamma \geq 0, \forall \boldsymbol{\vartheta} \in \mathscr{W}
$$

hence $\partial_{\delta} \mathscr{L}^{\mathcal{F}}-\boldsymbol{\lambda}=0$, so equation (19) holds (if the derivative is defined).

$(\Rightarrow)$

Lets prove the uniqueness of the minimum of the augmented Lagrangian $\mathscr{L}$, i.e. $\exists !(\boldsymbol{u}, \boldsymbol{\delta}, \boldsymbol{\lambda}) \in \mathscr{V} \times \mathscr{W} \times \mathscr{X}$ that verifies relations (18), with $\boldsymbol{u}$ the unique solution of the direct problem (7).

Taking an element $(\boldsymbol{u}, \boldsymbol{\delta}, \boldsymbol{\lambda}) \in \mathscr{V} \times \mathscr{W} \times \mathscr{X}$ with $\boldsymbol{u} \in \mathscr{V}, \llbracket \boldsymbol{u} \rrbracket \in \mathscr{W}$ minimum of $\mathscr{L}^{\boldsymbol{u}}$. Defining $\boldsymbol{\delta}=J \boldsymbol{u} \in \mathscr{W}$, the left inequality in (18) is accomplished $\forall \boldsymbol{\mu} \in \mathscr{X}$. If $\boldsymbol{u}$ is the minimum of the direct problem, from (17) and (22) it can be shown that the relation $\int_{\Gamma} \boldsymbol{\lambda} \cdot \llbracket \boldsymbol{v} \rrbracket d \Gamma=0, \quad \forall \boldsymbol{v} \in \mathscr{V}$ and $\int_{\Gamma} \boldsymbol{\lambda} \cdot \boldsymbol{p} d \Gamma \leq 0$ is satisfied $\forall \boldsymbol{p} \in \mathscr{W}$ because $\boldsymbol{\lambda}$ and $\boldsymbol{p}$ have the same direction but opposite senses. Then:

$$
\begin{aligned}
& \mathscr{L}^{\boldsymbol{u}}(\boldsymbol{u}) \leq \mathscr{L}^{\boldsymbol{u}}(\boldsymbol{v}), \forall \boldsymbol{v} \in \mathscr{V}, \\
& \mathscr{L}^{\boldsymbol{u}}(\boldsymbol{u}) \leq \mathscr{L}^{\boldsymbol{u}}(\boldsymbol{v})+\int_{\Gamma} \boldsymbol{\lambda} \cdot(\llbracket \boldsymbol{v} \rrbracket-\boldsymbol{p}) d \Gamma, \forall(\boldsymbol{v}, \boldsymbol{p}) \in \mathscr{V} \times \mathscr{W} .
\end{aligned}
$$

So the right inequality expressed like (21) also holds .

Observe that the equations (20), (22) and (24) are equivalent to the stationary point stated in (14) when the solution is reached. Relations (19), assign a physical meaning to the mathematical entity represented by the Lagrange multipliers both, for contact and cohesive regimes. The performance of discrete spaces is checked by numerical experiments for particular discretizations considering the inf-sup condition (Brezzi 1974). A rigorous proof of this aspect is beyond the scope of the present paper. A discussion about the properties of discrete spaces to fulfil stability conditions can be found in Baiges et al. (2012).

\section{FINITE ELEMENT DISCRETIZATION AND TAYLOR EXPANSION}

The numerical implementation of the formulation previously developed is presented in this section.

\section{Spatial discretization}

Let $\left\{\Omega_{h}\right\}_{h>0}$ be a mesh family that converges exactly to $\Omega$, where $h>0$ is the characteristic element size, and $\left\{\Gamma_{h}\right\}_{h>0}$ the set of mesh boundary that converges exactly to $\Gamma$. The spaces $\mathscr{V}_{h}, \mathscr{W}_{h} \mathrm{y} \mathscr{X}_{h}$ are the approximations to the continuum spaces.

Motivated by the decomposition coordination method, the approximation of the jump is non conforming, so $\mathscr{W}_{h} \nsubseteq \mathscr{W}$ in general does not hold due to the use of a discontinuous finite element discretization. The functional $\mathscr{G}_{0}$ in equation (14) is solved with fixed values of $\boldsymbol{\lambda}$ and $\boldsymbol{u}$, resulting $n_{\Gamma} \times n_{c}$ independent equations, where $n_{\Gamma}$ is the number of interface elements and $n_{c}$ the number of collocation points or quadrature points such that $\boldsymbol{\delta}_{h} \in \operatorname{ker} \mathscr{G}_{0}\left(\boldsymbol{u}_{h} ; \boldsymbol{\lambda}_{h}\right) \subset \mathscr{W}_{h}$. Then, the space $\mathscr{W}_{h}$ can be defined as:

$$
\mathscr{W}_{h}=\mathcal{P}_{d}^{k}\left(\Gamma_{h}\right)=\left\{\boldsymbol{\delta}_{h} \in \boldsymbol{L}^{2}\left(\Gamma_{h}\right): \forall \mathcal{I} \in \Gamma_{h},\left.\boldsymbol{\delta}_{h}\right|_{\mathcal{I}} \in \mathbb{P}_{k} \wedge \boldsymbol{\delta}_{h} \cdot \boldsymbol{n} \geq 0\right\} \not \subset \mathscr{W},
$$

where $\mathbb{P}_{k}$ is the polynomial space with order less or equal to $k, \mathcal{I}$ is a discrete element in $\Gamma_{h}$ and the sub index $d$ represents a discontinuous interpolation. The discrete conforming spaces for displacements and Lagrange multipliers are stated as follows:

$$
\begin{gathered}
\mathscr{V}_{h}=\mathcal{P}_{c}^{k}\left(\Omega_{h}\right)=\left\{\boldsymbol{v}_{h} \in C^{0}\left(\Omega_{h}\right): \forall \mathcal{E} \in \Omega_{h},\left.\left.\boldsymbol{v}_{h}\right|_{\mathcal{E}} \in \mathbb{P}_{k} \wedge \boldsymbol{v}_{h}\right|_{\Gamma_{h}^{D}}=0\right\} \subset \mathscr{V}, \\
\mathscr{X}_{h}=\mathcal{P}_{c}^{k}\left(\Gamma_{h}\right)=\left\{\boldsymbol{\lambda}_{h} \in \boldsymbol{C}^{0}\left(\Gamma_{h}\right): \forall \mathcal{I} \in \Gamma_{h},\left.\boldsymbol{\lambda}_{h}\right|_{\mathcal{I}} \in \mathbb{P}_{k}\right\} \subset \mathscr{X} .
\end{gathered}
$$

The discrete sets (26) and (27) are assumed to be $\mathscr{V}_{h}=\mathcal{P}_{c}^{2}\left(\Omega_{h}\right)$ and $\mathscr{X}_{h}=\mathcal{P}_{c}^{1}\left(\Gamma_{h}\right)$ respectively, to fulfil the inf-sup condition (Brezzi 1974). The resulting discretization is represented schematically in figure 3 for the two dimensional case. The continuum fields can be expressed in a discrete way:

$$
\boldsymbol{u}(\boldsymbol{x}, t) \approx \boldsymbol{u}^{h}=[\mathbb{N}(\boldsymbol{x}, t)]\{\boldsymbol{U}\} \Rightarrow \delta \boldsymbol{u} \approx \delta \boldsymbol{u}^{h}=[\mathbb{N}(\boldsymbol{x}, t)]\{\delta \boldsymbol{U}\}
$$




$$
\begin{gathered}
\boldsymbol{\varepsilon}(\boldsymbol{x}, t)=\nabla^{s} \boldsymbol{u} \approx \nabla^{s} \boldsymbol{u}^{h}=\left[\nabla^{s} \mathbb{N}(\boldsymbol{x}, t)\right] \cdot\{\boldsymbol{U}\}, \\
\boldsymbol{\lambda}(\boldsymbol{x}, t) \approx \boldsymbol{\lambda}^{h}=[\mathbb{L}(\boldsymbol{s}, t)] \cdot\{\boldsymbol{\Lambda}\} \Rightarrow \delta \boldsymbol{\lambda} \approx \delta \boldsymbol{\lambda}^{h}=[\mathbb{L}(\boldsymbol{s}, t)] \cdot\{\delta \boldsymbol{\Lambda}\},
\end{gathered}
$$

being $\mathbb{N}(\boldsymbol{x}, t)$ and $\mathbb{L}(s, t)$ the interpolation matrices for displacements and Lagrange multipliers respectively. The displacement jump is approximated with the matrix $\mathbb{J}(s, t)=\breve{\mathbb{N}}_{+}(s, t)-\breve{\mathbb{N}}_{-}(s, t)$ called jump operator that maps the displacement field $\boldsymbol{u}$ to the gap field $\llbracket \boldsymbol{u} \rrbracket$ :

$$
\llbracket \boldsymbol{u}(\boldsymbol{s}, t) \rrbracket \approx \llbracket \boldsymbol{u}^{h} \rrbracket=\left[\breve{\mathbb{N}}_{+}(\boldsymbol{s}, t)-\breve{\mathbb{N}}_{-}(\boldsymbol{s}, t)\right] \cdot\{\boldsymbol{U}\}=[\mathbb{J}(\boldsymbol{s}, t)] \cdot\{\boldsymbol{U}\} \Rightarrow \llbracket \delta \boldsymbol{u} \rrbracket \approx \llbracket \delta \boldsymbol{u}^{h} \rrbracket=[\mathbb{J}(\boldsymbol{s}, t)] \cdot\{\delta \boldsymbol{U}\}
$$

where $\breve{\mathbb{N}}_{+}$and $\breve{\mathbb{N}}_{-}$are computed as the bulk shape functions particularized in both sides of the crack lips.

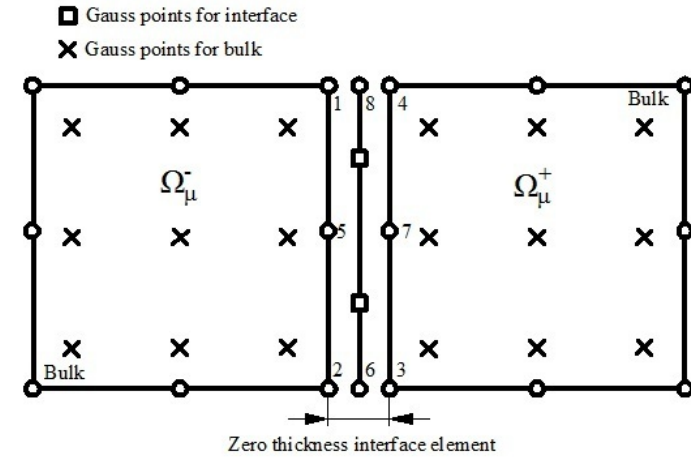

(a) Finite element used for Q8/L2 interpolation.

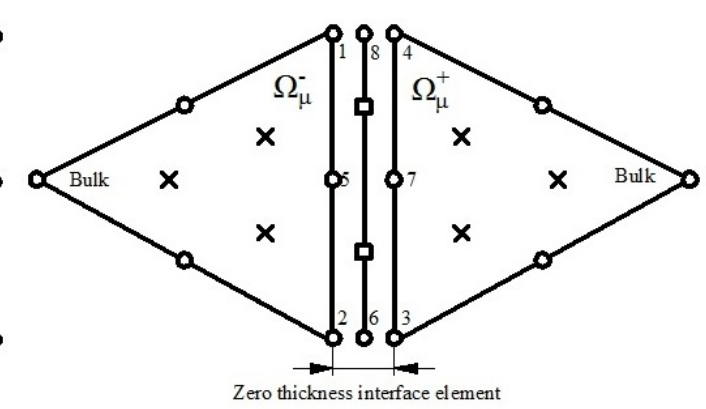

(b) Finite element used for T6/L2 interpolation.

Figure 3. Finite element discretization used.

\section{Pseudo-temporal discretization}

A Taylor expansion of the Functionals $\mathscr{G}_{1}$ and $\mathscr{G}_{2}$ is developed for the implementation in a classical Newton Raphson solver:

$$
\mathscr{G}_{i}\left(\left.\boldsymbol{u}\right|_{n+1} ;\left.\boldsymbol{\lambda}\right|_{n+1}\right)=\mathscr{G}_{i}\left(\left.\boldsymbol{u}\right|_{n} ;\left.\boldsymbol{\lambda}\right|_{n}\right)+\left.\overrightarrow{\partial_{\boldsymbol{u}} \mathscr{G}_{i}}\right|_{n}+\left.\overrightarrow{\partial_{\boldsymbol{\lambda}} \mathscr{G}_{i}}\right|_{n}+\mathscr{H}
$$

with $1 \leq i \leq 2$. The higher order terms $\mathscr{H}$ in equation (32) are neglected, and the directional derivatives $\overrightarrow{\partial \boldsymbol{u}}_{i}$ and $\overrightarrow{\partial \boldsymbol{\lambda}}_{i}$ are computed as follows:

$$
\vec{\partial}_{\bullet} \mathscr{G}_{i}=\lim _{t \rightarrow 0^{+}} \frac{1}{t}\left[\mathscr{G}_{i}(\bullet+t \delta \bullet)-\mathscr{G}_{i}(\bullet)\right]=\partial_{\bullet} \mathscr{G}_{i} \cdot \delta \bullet, \quad \text { with } \bullet=\boldsymbol{u}, \boldsymbol{\lambda} .
$$

Replacing $\mathscr{G}_{1}$ and $\mathscr{G}_{2}$ in equation (33), the following directional derivatives are obtained:

$$
\begin{aligned}
\overrightarrow{\partial_{\boldsymbol{u}} \mathscr{G}_{1}} & =\int_{\Omega \backslash \Gamma} \nabla^{s} \delta \boldsymbol{u}: \boldsymbol{C}^{t}: \nabla^{s} \delta \boldsymbol{u} d \Omega+\int_{\Gamma} \gamma\left(\llbracket \delta \boldsymbol{u} \rrbracket-\gamma \frac{\partial \boldsymbol{\delta}}{\partial \boldsymbol{t}} \cdot \llbracket \delta \boldsymbol{u} \rrbracket\right) \cdot \llbracket \delta \boldsymbol{u} \rrbracket d \Gamma, \\
\overrightarrow{\partial_{\boldsymbol{\lambda}} \mathscr{G}_{1}} & =\int_{\Gamma}\left[\delta \boldsymbol{\lambda}-\gamma \frac{\partial \boldsymbol{\delta}}{\partial \boldsymbol{t}} \cdot \delta \boldsymbol{\lambda}\right] \cdot \llbracket \delta \boldsymbol{u} \rrbracket d \Gamma, \\
\overrightarrow{\partial_{\boldsymbol{u}} \mathscr{G}_{2}} & =\int_{\Gamma}\left[\llbracket \delta \boldsymbol{u} \rrbracket-\gamma \frac{\partial \boldsymbol{\delta}}{\partial \boldsymbol{t}} \cdot \llbracket \delta \boldsymbol{u} \rrbracket\right] \cdot \delta \boldsymbol{\lambda} d \Gamma, \\
\overrightarrow{\partial_{\boldsymbol{\lambda}} \mathscr{G}_{2}} & =-\int_{\Gamma} \delta \boldsymbol{\lambda} \cdot\left[\frac{\partial \boldsymbol{\delta}}{\partial \boldsymbol{t}} \cdot \delta \boldsymbol{\lambda}\right] d \Gamma .
\end{aligned}
$$

Substituting equations (34)-(37) in equation (32), a residual $\mathscr{R}$ can be defined as:

$$
\mathscr{R}\left(\left.\boldsymbol{u}\right|_{n+1} ;\left.\boldsymbol{\lambda}\right|_{n+1}\right)=\left\{\begin{array}{c}
\left.\mathscr{G}_{1}\right|_{n+1}-\left.\mathscr{G}_{1}\right|_{n} \\
\left.\mathscr{G}_{2}\right|_{n+1}-\left.\mathscr{G}_{2}\right|_{n}
\end{array}\right\}=\left.\left.\left[\begin{array}{cc}
\partial_{\boldsymbol{u}} \mathscr{G}_{1} & \partial_{\boldsymbol{\lambda}} \mathscr{G}_{1} \\
\partial_{\boldsymbol{u}} \mathscr{G}_{2} & \partial_{\boldsymbol{\lambda}} \mathscr{G}_{2}
\end{array}\right]\right|_{n} \cdot\left\{\begin{array}{l}
\delta \boldsymbol{u} \\
\delta \boldsymbol{\lambda}
\end{array}\right\}\right|_{n+1}+\mathscr{H} .
$$

By substituting equations (28) to (31) into equation (38), the following relation is obtained: 


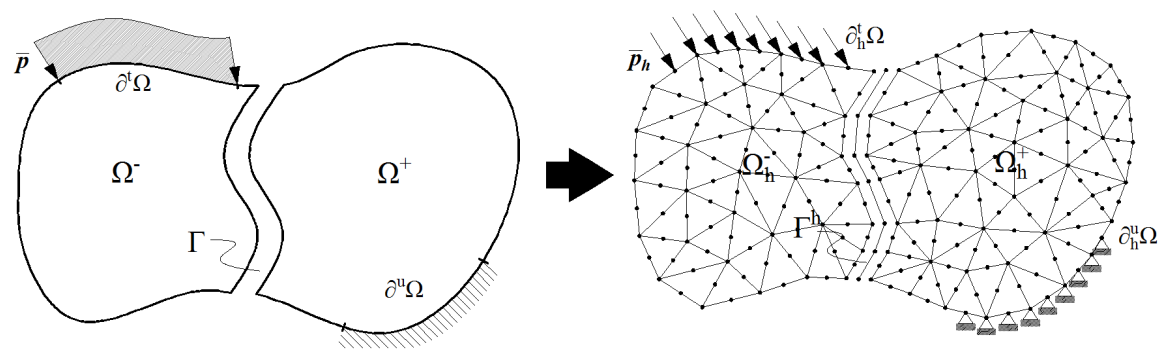

Figure 4. Domain decomposition of $\Omega$ considering a cohesive fracture $\Gamma$.

$$
\mathbb{R}\left(\left.\boldsymbol{u}\right|_{n+1} ;\left.\boldsymbol{\lambda}\right|_{n+1}\right)=\left.\left.\left[\begin{array}{ll}
\mathbb{K}_{\boldsymbol{u u}} & \mathbb{K}_{\boldsymbol{u \lambda}} \\
\mathbb{K}_{\boldsymbol{\lambda} \boldsymbol{u}} & \mathbb{K}_{\boldsymbol{\lambda \boldsymbol { \lambda }}}
\end{array}\right]\right|_{n}\left\{\begin{array}{c}
\delta \boldsymbol{U} \\
\delta \boldsymbol{\Lambda}
\end{array}\right\}\right|_{n+1}=\left.\left.[\mathbb{K}]\right|_{n}\{\delta \boldsymbol{X}\}\right|_{n+1}
$$

The stiffness matrix is $\mathbb{K}$ splitted in an initial matrix $\mathbb{K}^{0}$ (computed during the pre process of the FEM solver), and $\mathbb{K}^{T}$ (updated in each iteration), as follows

$$
\mathbb{K}=\mathbb{K}^{0}+\mathbb{K}^{T}=\left[\begin{array}{cc}
\mathbb{K}_{\boldsymbol{u} \boldsymbol{u}}^{0} & \mathbb{K}_{\boldsymbol{u \lambda}}^{0} \\
\mathbb{K}_{\boldsymbol{\lambda} \boldsymbol{u}}^{0} & \mathbb{K}_{\boldsymbol{\lambda} \boldsymbol{\lambda}}^{0}
\end{array}\right]+\left[\begin{array}{cc}
\mathbb{K}_{\boldsymbol{u} \boldsymbol{u}}^{T} & \mathbb{K}_{\boldsymbol{u \lambda}}^{T} \\
\mathbb{K}_{\boldsymbol{\lambda} \boldsymbol{u}}^{T} & \mathbb{K}_{\boldsymbol{\lambda} \boldsymbol{\lambda}}^{T}
\end{array}\right]
$$

Using the notation defined in Figure 4, the sub-matrices of the initialization matrix $\mathbb{K}^{0}$, are explicitly expressed as follows:

$$
\begin{aligned}
\mathbb{K}_{\boldsymbol{u} \boldsymbol{u}}^{0} & =\int_{\Omega^{h} \backslash \Gamma^{h}}\left[\nabla^{s} \mathbb{N}(\boldsymbol{x}, t)\right]^{\boldsymbol{T}}\left[\mathbb{C}^{t}\right]\left[\nabla^{s} \mathbb{N}(\boldsymbol{x}, t)\right] d \Omega^{h}+\int_{\Gamma^{h}} \gamma[\mathbb{J}(\boldsymbol{s}, t)]^{\boldsymbol{T}}[\mathbb{J}(\boldsymbol{s}, t)] d \Gamma^{h}, \\
\mathbb{K}_{\boldsymbol{u} \boldsymbol{\lambda}}^{0} & =\int_{\Gamma^{h}}[\mathbb{L}(\boldsymbol{s}, t)]^{\boldsymbol{T}}[\mathbb{J}(\boldsymbol{s}, t)] d \Gamma^{h} \\
\mathbb{K}_{\boldsymbol{\lambda} \boldsymbol{u}}^{0} & =\int_{\Gamma^{h}}[\mathbb{J}(\boldsymbol{s}, t)]^{\boldsymbol{T}}[\mathbb{L}(\boldsymbol{s}, t)] d \Gamma^{h} \\
\mathbb{K}_{\boldsymbol{\lambda} \boldsymbol{\lambda}}^{0} & =\Theta
\end{aligned}
$$

Observe that the above components of the stiffness matrix are fixed in the overall simulation and the sub matrices of $\mathbb{K}^{T}$ depending on $\frac{\partial \boldsymbol{\delta}}{\partial \boldsymbol{t}}$, must be updated in each iteration as follows:

$$
\begin{aligned}
\mathbb{K}_{\boldsymbol{u} \boldsymbol{u}}^{T} & =-\int_{\Gamma^{h}} \gamma^{2}[\mathbb{J}(\boldsymbol{s}, t)]^{\boldsymbol{T}}\left[\frac{\partial \boldsymbol{\delta}}{\partial \boldsymbol{t}}\right][\mathbb{J}(\boldsymbol{s}, t)] d \Gamma^{h} \\
\mathbb{K}_{\boldsymbol{u \boldsymbol { \lambda }}}^{T} & =-\int_{\Gamma^{h}} \gamma[\mathbb{L}(\boldsymbol{s}, t)]^{\boldsymbol{T}}\left[\frac{\partial \boldsymbol{\delta}}{\partial \boldsymbol{t}}\right][\mathbb{J}(\boldsymbol{s}, t)] d \Gamma^{h}, \\
\mathbb{K}_{\boldsymbol{\lambda} \boldsymbol{u}}^{T} & =-\int_{\Gamma^{h}} \gamma[\mathbb{J}(\boldsymbol{s}, t)]^{\boldsymbol{T}}\left[\frac{\partial \boldsymbol{\delta}}{\partial \boldsymbol{t}}\right][\mathbb{L}(\boldsymbol{s}, t)] d \Gamma^{h}, \\
\mathbb{K}_{\boldsymbol{\lambda} \boldsymbol{\lambda}}^{T} & =-\int_{\Gamma^{h}}[\mathbb{L}(\boldsymbol{s}, t)]^{\boldsymbol{T}}\left[\frac{\partial \boldsymbol{\delta}}{\partial \boldsymbol{t}}\right][\mathbb{L}(\boldsymbol{s}, t)] d \Gamma^{h}
\end{aligned}
$$

\section{TRACTION SEPARATION LAW}

An exponential cohesive zone model with a linear unloading based on the simplification of the potential proposed by Park et al. (2009) is implemented in this paper. The potential is defined as follow:

$$
\psi\left(\delta_{e q}\right)=\left\{\begin{array}{ll}
G_{c}\left(1-\left(1-\frac{\delta_{e q}}{\delta_{c}}\right)^{\beta}\right), & \text { if } \delta_{e q} \leq \delta_{c} \\
G_{c}, & \text { if } \delta_{e q}>\delta_{c}
\end{array} .\right.
$$

The parameter $\beta$ defines the traction-separation function shape in normal and tangential directions as shown figure 5. The parameter $G_{c}=\frac{1}{\beta} \sigma_{c} \delta_{c}$ is used to denote the fracture energy, $\sigma_{c}$ is the critical tension and $\delta_{c}$ is the critical displacement. The potential is totally defined when three of the four parameters $\left(G_{c}, \sigma_{c}, \delta_{c}\right.$ and $\left.\beta\right)$, are fixed. The cohesive forces in the crack are driven by an equivalent displacement:

$$
\delta_{e q}=\|\boldsymbol{\delta}\|=\sqrt{\boldsymbol{\delta} \cdot \boldsymbol{\delta}}
$$




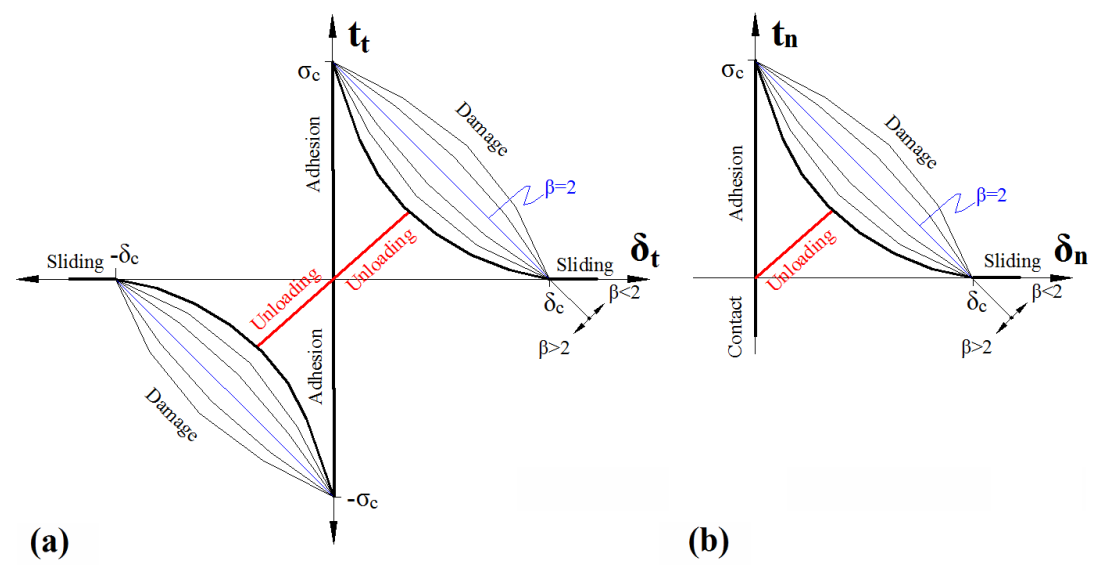

Figure 5. Traction separation law based on the potential by Park et al. (2009) for $\delta_{n}>0$. a) Tangential law b) Normal law.

The sub-gradient of $\Psi$ should be defined to fix the constitutive behaviour in each collocation point. The procedure proposed in Clarke (1978) and Clarke (1990) is used for this purpose, where $\partial_{\delta} \Psi$ is given by

$$
\begin{gathered}
\partial_{\boldsymbol{\delta}} \Psi(\boldsymbol{\delta}, \kappa)=\left\{\boldsymbol{t} \in \boldsymbol{H}^{-\frac{1}{2}} \mid \forall \boldsymbol{v} \in \boldsymbol{H}^{\frac{1}{2}}, \boldsymbol{t} \cdot \boldsymbol{v} \leq \Psi^{0}(\boldsymbol{\delta}, \kappa, \boldsymbol{v})\right\}, \text { with fixed } \kappa, \\
\Psi^{0}(\boldsymbol{\delta}, \kappa, \boldsymbol{v})=\limsup _{\boldsymbol{q} \rightarrow \boldsymbol{\delta} ; \zeta \rightarrow 0^{+}} \frac{\Psi(\boldsymbol{q}+\zeta \boldsymbol{v}, \kappa)-\Psi(\boldsymbol{q}, \kappa)}{\zeta}, \text { with fixed } \kappa .
\end{gathered}
$$

Given a direction $\boldsymbol{v} \in \boldsymbol{H}^{\frac{1}{2}}$, the generalized directional derivative $\Psi^{0}$ can be defined with equation (52). It is clear that these concepts are applicable to the constitutive modes where $\Psi$ is not differentiable, corresponding to adhesion, unloading and damage. In other cases, the sub gradient contains just one element $t=\frac{\partial \Psi}{\partial \boldsymbol{\delta}}$.

An irreversible variable $\kappa$ is introduced to control the crack opening/closure:

$$
\kappa(t)=\sup _{t^{\prime}<t^{*}} \delta_{e q}\left(t^{\prime}\right)
$$

where $t^{*}$ is the actual time. Due to the definition of the pseudo potential $\Psi$ (Lorentz 2008), the following restriction must be included to fulfil the double inequality (11):

$$
t_{n-} \leq 0, \quad \delta_{n} \geq 0, \quad \text { and } t_{n-} \cdot \delta_{n}=0
$$

where $t_{n-}=\langle\boldsymbol{t} \cdot \boldsymbol{n}\rangle_{-}$is the negative part (inverse Macauly brackets) of the projection of $\boldsymbol{t}$ over the normal vector $\boldsymbol{n}$ measured in the interface local coordinates. The present theoretical framework can be interpreted in terms of classical contact formulations.

If equation (12) is solved as a coupled problem, the supplementary variable should be considered as an additional variable. When the coordination-decomposition method is used to transform a global non-differential problem into a set of local algebraic problems, an uncoupled explicit-time integration in collocation points to calculate supplementary variable is carried out. A staggered solution is obtained and the desired function $\boldsymbol{\delta}_{h}$ can be updated as:

$$
\begin{gathered}
\boldsymbol{\delta}_{h}^{n+1}=\arg \min _{\boldsymbol{\delta}_{h} \in \boldsymbol{H}^{\frac{1}{2}}}\left\{\boldsymbol{\lambda}_{h}^{n} \cdot\left(\llbracket \boldsymbol{u}_{h}^{n} \rrbracket-\boldsymbol{\delta}_{h}^{n}\right)+\frac{\gamma}{2}\left(\llbracket \boldsymbol{u}_{h}^{n} \rrbracket-\boldsymbol{\delta}_{h}^{n}\right)^{2}+\Psi\left(\boldsymbol{\delta}_{h}, \kappa^{n}\right)\right\} \\
\kappa^{n+1}=\max \left(\kappa^{n}, \delta_{e q}^{n+1}\right)
\end{gathered}
$$

with $(\bullet)^{n+1}$ representing the actual variable. Performing the directional derivative that makes stationary the functional $\mathscr{L}$ in the direction of $\boldsymbol{\delta}$ in each collocation point, the discrete set of kinematically admissible supplementary variable is given:

$$
\boldsymbol{\delta}_{h}^{n+1}=\left\{\boldsymbol{\delta} \in \boldsymbol{H}^{\frac{1}{2}} \mid\left[\boldsymbol{\lambda}^{n}+\gamma \llbracket \boldsymbol{u}^{n} \rrbracket-\gamma \boldsymbol{\delta}_{h}^{n}\right] \in \partial_{\boldsymbol{\delta}} \Psi\left(\boldsymbol{\delta}, \kappa^{n}\right)\right\} .
$$

The notation and the details about the sub gradient computation are given in the following section.

\section{Computation of the explicit sub-gradient.}

The sub gradients for each load stage of the constitutive model is computed in this section: 


\section{Notation}

The unit normal vector is denoted as $\boldsymbol{n}$ and $\boldsymbol{m}$ is the unitary tangent vector, both defined in the interface local reference system:

$$
\boldsymbol{n} \cdot \boldsymbol{n}=1, \quad \boldsymbol{m} \cdot \boldsymbol{m}=1, \quad \boldsymbol{n} \cdot \boldsymbol{m}=0 .
$$

The projection of $\boldsymbol{v}$ onto the vector $\boldsymbol{n}$ denoted $v_{n} \in \mathbb{R}$ is computed as:

$$
v_{n}=\boldsymbol{v} \cdot \boldsymbol{n},
$$

and the component of a vector $\boldsymbol{v}$ onto the vector $\boldsymbol{m}$ denoted $\boldsymbol{v}_{\|}$is:

$$
\boldsymbol{v}_{\|}=\boldsymbol{v}-v_{n} \boldsymbol{n}=(\mathbb{I}-\boldsymbol{n} \otimes \boldsymbol{n}) \boldsymbol{v} .
$$

Vector $\boldsymbol{v}$ with positive part of the normal component noted $\langle\boldsymbol{v}\rangle_{+}$is:

$$
\langle\boldsymbol{v}\rangle_{+}=\left\langle v_{n}\right\rangle \boldsymbol{n}+\boldsymbol{v}_{\|},
$$

where $\langle\bullet\rangle$ are the Macaulay brackets and equipped with the norm:

$$
\|\boldsymbol{v}\|_{+}=\left(\langle\boldsymbol{v}\rangle_{+} \cdot\langle\boldsymbol{v}\rangle_{+}\right)^{\frac{1}{2}}
$$

\section{Sub-gradient computation.}

Depending on the loading zone the sub-gradient can be defined as follows:

\section{- Adhesion}

The discontinuity is under perfect adhesion (the initiation criteria is non activated). When $\delta=0$ and $\kappa=0$, the directional derivative in the direction of vector $\boldsymbol{t}$ becomes:

$$
\partial_{\boldsymbol{\delta}} \Psi(\boldsymbol{\delta}, \kappa)=\left\{\boldsymbol{t} \in \boldsymbol{H}^{-\frac{1}{2}} \mid\|\boldsymbol{t}\|_{+} \leq \sigma_{c}\right\}
$$

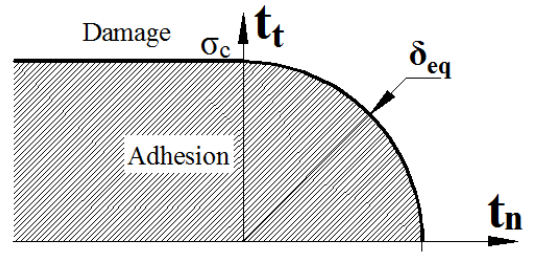

Figure 6. Initiation criteria implemented in the formulation (Lorentz 2008).

Figure 6 shows a graphical interpretation of the set exposed in Equation (63) plotted in the normal and tangential projection of $t$.

- Unloading

The unloading is activated when $\delta_{e q} \leq \kappa$ with $\kappa>0$. The sub-gradient of $\Psi$ is defined as follows:

$$
\partial_{\boldsymbol{\delta}} \Psi(\boldsymbol{\delta}, \kappa)=\left\{t_{n} \boldsymbol{n}+\rho \boldsymbol{\delta} \mid t_{n} \leq 0 \wedge \delta_{n} \geq 0 \wedge t_{n} \delta_{n}=0\right\} .
$$

where $\rho=\frac{\psi^{\prime}(\kappa)}{\kappa}$.Considering $t_{n} \leq 0$ and $\delta_{n} \geq 0$ with Equation (57) and $\tilde{\boldsymbol{t}}=\boldsymbol{\lambda}+\gamma \llbracket \boldsymbol{u} \rrbracket$

$$
\langle\tilde{\boldsymbol{t}}\rangle_{+}=\gamma \boldsymbol{\delta}+\rho \boldsymbol{\delta} \Rightarrow \boldsymbol{\delta}=\frac{\langle\tilde{\boldsymbol{t}}\rangle_{+}}{(\gamma+\rho)} .
$$

Combining (64) and (65), the following expression is obtained for $\delta$ :

$$
\boldsymbol{\delta}=\frac{1}{\gamma+\frac{\psi^{\prime}(\kappa)}{\kappa}}\langle\tilde{\boldsymbol{t}}\rangle_{+} .
$$

Considering $t_{n}=0$ and $\delta_{n}>0$ :

$$
\frac{\partial \boldsymbol{\delta}}{\partial \tilde{\boldsymbol{t}}}=\frac{1}{\gamma+\frac{\psi^{\prime}(\kappa)}{\kappa}} \frac{\partial}{\partial \tilde{\boldsymbol{t}}} \tilde{\boldsymbol{t}}=\frac{1}{\gamma+\frac{\psi^{\prime}(\kappa)}{\kappa}} \mathbb{I}
$$


if $t_{n}<0$ and $\delta_{n}=0$ :

$$
\frac{\partial \boldsymbol{\delta}}{\partial \tilde{\boldsymbol{t}}}=\frac{1}{\gamma+\frac{\psi^{\prime}(\kappa)}{\kappa}} \frac{\partial}{\partial \tilde{\boldsymbol{t}}} \tilde{\boldsymbol{t}}_{\|}=\frac{1}{\gamma+\frac{\psi^{\prime}(\kappa)}{\kappa}}(\mathbb{I}-\boldsymbol{n} \otimes \boldsymbol{n})
$$

The activation criteria for the unloading stage, can be expressed in a more convenient way:

$$
\delta_{e q}<\kappa \Rightarrow\|\boldsymbol{\delta}\|_{+}=\delta_{e q}=\frac{1}{\gamma+\frac{\psi^{\prime}(\kappa)}{\kappa}}\|\tilde{\boldsymbol{t}}\|_{+} \leq \kappa \Rightarrow\|\tilde{\boldsymbol{t}}\|_{+} \leq \kappa \gamma+\psi^{\prime}(\kappa) .
$$

- Damage

If $\delta_{e q}>\kappa$, the crack is propagating. The sub gradient is defined as:

$$
\partial_{\boldsymbol{\delta}} \Psi(\boldsymbol{\delta}, \kappa)=\left\{t_{n} \boldsymbol{n}+\rho \boldsymbol{\delta} \mid t_{n} \leq 0 \wedge \delta_{n} \geq 0 \wedge t_{n} \delta_{n}=0\right\} .
$$

In order to explicitly obtain $\delta$, let consider $\rho=\frac{\psi^{\prime}\left(\delta_{e q}\right)}{\delta_{e q}}$ together with the equation (65):

$$
\begin{aligned}
\langle\tilde{\boldsymbol{t}}\rangle_{+} & =\left(\frac{\psi^{\prime}\left(\delta_{e q}\right)}{\delta_{e q}}+\gamma\right)\langle\boldsymbol{\delta}\rangle_{+} \Rightarrow\|\tilde{\boldsymbol{t}}\|_{+}=\left(\frac{\psi^{\prime}\left(\delta_{e q}\right)}{\delta_{e q}}+\gamma\right) \delta_{e q}, \\
\|\tilde{\boldsymbol{t}}\|_{+} & =\psi^{\prime}\left(\delta_{e q}\right)+\gamma \delta_{e q}
\end{aligned}
$$

From equation (65) and considering the last constraints:

$$
\boldsymbol{\delta}=\delta_{e q} \frac{\langle\tilde{\boldsymbol{t}}\rangle_{+}}{\|\tilde{\boldsymbol{t}}\|_{+}}
$$

where $\delta_{e q}$ is obtained from the equation (71).

To get the derivatives of $\boldsymbol{\delta}$, let first consider $t_{n}=0, \delta_{n}>0$ and $\delta_{e q} \leq \delta_{c}$ :

$$
\begin{aligned}
\frac{\partial \boldsymbol{\delta}}{\partial \tilde{\boldsymbol{t}}} & =\frac{\delta_{e q}}{\|\tilde{\boldsymbol{t}}\|_{+}^{2}}\left[\|\tilde{\boldsymbol{t}}\|_{+} \frac{\partial}{\partial \tilde{\boldsymbol{t}}}\langle\tilde{\boldsymbol{t}}\rangle_{+}-\langle\tilde{\boldsymbol{t}}\rangle_{+} \otimes \frac{\langle\tilde{\boldsymbol{t}}\rangle_{+}}{\|\tilde{\boldsymbol{t}}\|_{+}}\right]+\left[\frac{\partial}{\partial \tilde{\boldsymbol{t}}} \delta_{e q}\right] \otimes \frac{\langle\tilde{\boldsymbol{t}}\rangle_{+}}{\|\tilde{\boldsymbol{t}}\|_{+}}= \\
& =\frac{\delta_{e q}}{\|\tilde{\boldsymbol{t}}\|_{+}}\left[\mathbb{I}-\frac{\langle\tilde{\boldsymbol{t}}\rangle_{+}}{\|\tilde{\boldsymbol{t}}\|_{+}} \otimes \frac{\langle\tilde{\boldsymbol{t}}\rangle_{+}}{\|\tilde{\boldsymbol{t}}\|_{+}}\right]+\left[\frac{\partial}{\partial \tilde{\boldsymbol{t}}} \delta_{e q}\right] \otimes \frac{\langle\tilde{\boldsymbol{t}}\rangle_{+}}{\|\tilde{\boldsymbol{t}}\|_{+}}
\end{aligned}
$$

When $\delta_{e q}>\delta_{c}$ :

$$
\delta_{e q}=\frac{\|\boldsymbol{t}\|}{\gamma} \Rightarrow \frac{\partial \boldsymbol{\delta}}{\partial \tilde{\boldsymbol{t}}}=\frac{\partial}{\partial \tilde{\boldsymbol{t}}}\left[\frac{\|\boldsymbol{t}\|}{\gamma} \frac{\tilde{\boldsymbol{t}}}{\|\tilde{\boldsymbol{t}}\|}\right]=\frac{1}{\gamma} \mathbb{I} .
$$

Now, considering $t_{n}<0, \delta_{n}=0$ and $\delta_{e q} \leq \delta_{c}$ :

$$
\begin{aligned}
\frac{\partial \boldsymbol{\delta}}{\partial \tilde{\boldsymbol{t}}} & =\left[\frac{\partial}{\partial \tilde{\boldsymbol{t}}} \delta_{e q}\right] \otimes \frac{\tilde{\boldsymbol{t}}_{\|}}{\left\|\tilde{\boldsymbol{t}}_{\|}\right\|}+\delta_{e q} \frac{\partial}{\partial \tilde{\boldsymbol{t}}}\left[\frac{\tilde{\boldsymbol{t}}_{\|}}{\left\|\tilde{\boldsymbol{t}}_{\|}\right\|}\right]=\left[\frac{\partial}{\partial \tilde{\boldsymbol{t}}} \delta_{e q}\right] \otimes \frac{\tilde{\boldsymbol{t}}_{\|}}{\left\|\tilde{\boldsymbol{t}}_{\|}\right\|}+\frac{\delta_{e q}}{\left\|\tilde{\boldsymbol{t}}_{\|}\right\|^{2}}\left[\left\|\tilde{\boldsymbol{t}}_{\|}\right\|\left(\frac{\partial}{\partial \boldsymbol{\delta}} \tilde{\boldsymbol{t}}_{\|}\right)-\tilde{\boldsymbol{t}}_{\|} \otimes \frac{\partial}{\partial \boldsymbol{\delta}}\left\|\tilde{\boldsymbol{t}}_{\|}\right\|\right] \\
& =\left[\frac{\partial}{\partial \tilde{\boldsymbol{t}}} \delta_{e q}\right] \otimes \frac{\tilde{\boldsymbol{t}}_{\|}}{\left\|\tilde{\boldsymbol{t}}_{\|}\right\|}+\frac{\delta_{e q}}{\left\|\tilde{\boldsymbol{t}}_{\|}\right\|^{2}}\left[(\mathbb{I}-\boldsymbol{n} \otimes \boldsymbol{n})\left\|\tilde{\boldsymbol{t}}_{\|}\right\|-\tilde{\boldsymbol{t}}_{\|} \otimes \frac{\tilde{\boldsymbol{t}}_{\|}}{\left\|\tilde{\boldsymbol{t}}_{\|}\right\|}(\mathbb{I}-\boldsymbol{n} \otimes \boldsymbol{n})\right] \\
& =\left[\frac{\partial}{\partial \tilde{\boldsymbol{t}}} \delta_{e q}\right] \otimes \frac{\tilde{\boldsymbol{t}}_{\|}}{\left\|\tilde{\boldsymbol{t}}_{\|}\right\|}+\frac{\delta_{e q}}{\left\|\tilde{\boldsymbol{t}}_{\|}\right\|}\left[(\mathbb{I}-\boldsymbol{n} \otimes \boldsymbol{n})-\frac{\tilde{\boldsymbol{t}}_{\|}}{\left\|\tilde{\boldsymbol{t}}_{\|}\right\|} \otimes \frac{\tilde{\boldsymbol{t}}_{\|}}{\left\|\tilde{\boldsymbol{t}}_{\|}\right\|}\right] \\
& =\left[\frac{\partial}{\partial \tilde{\boldsymbol{t}}} \delta_{e q}\right] \otimes \frac{\tilde{\boldsymbol{t}}_{\|}}{\left\|\tilde{\boldsymbol{t}}_{\|}\right\|}+\frac{\delta_{e q}}{\left\|\tilde{\boldsymbol{t}}_{\|}\right\|}[\mathbb{I}-\boldsymbol{n} \otimes \boldsymbol{n}-\boldsymbol{m} \otimes \boldsymbol{m}]=\left[\frac{\partial}{\partial \tilde{\boldsymbol{t}}} \delta_{e q}\right] \otimes \frac{\tilde{\boldsymbol{t}}_{\|}}{\left\|\tilde{\boldsymbol{t}}_{\|}\right\|} .
\end{aligned}
$$

if $\delta_{e q}>\delta_{c}$ :

$$
\frac{\partial \boldsymbol{\delta}}{\partial \tilde{\boldsymbol{t}}}=\frac{\partial}{\partial \tilde{\boldsymbol{t}}}\left[\frac{\left\|\tilde{\boldsymbol{t}}_{\|}\right\|}{\gamma} \frac{\tilde{\boldsymbol{t}}_{\|}}{\left\|\tilde{\boldsymbol{t}}_{\|}\right\|}\right]=\frac{1}{\gamma}(\mathbb{I}-\boldsymbol{n} \otimes \boldsymbol{n})
$$

The damage branch is activated when $\|\tilde{\boldsymbol{t}}\|_{+}>\kappa \gamma+\psi^{\prime}(\kappa)$. The equations are condensed in Algorithm 1. 


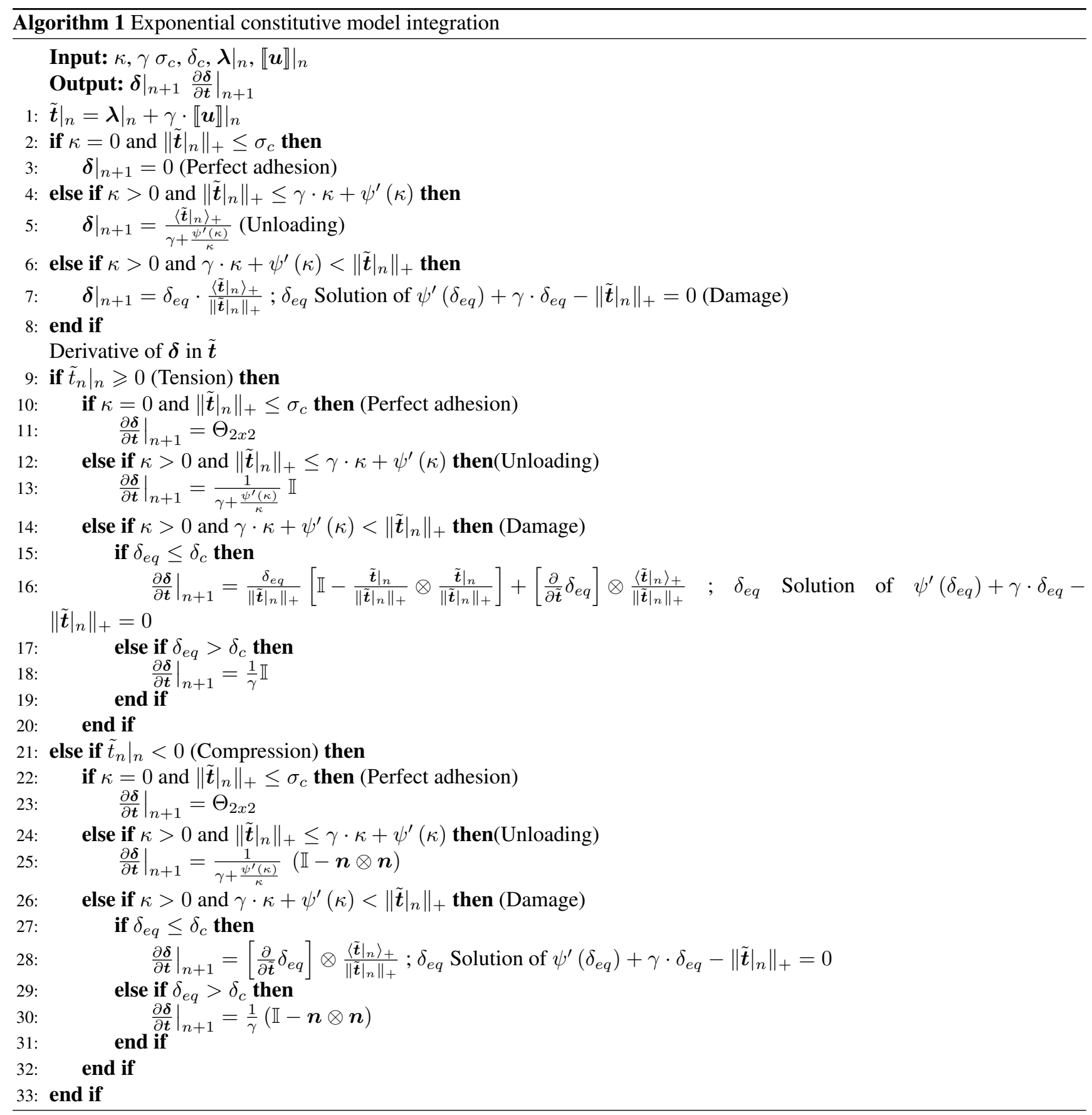

\section{An algorithm to insert Lagrangian zero-thickness interface elements.}

A new algorithm to insert Lagrange multipliers based zero-thickness interface elements is presented in this section. This technique is based on an extension of the method presented in Nguyen (2014) and represents an original contribution of this paper. For this case, additional nodes are generated to consider the numerical calculation of Lagrange multipliers in meso-mechanical problems.

Figure 7 shows the proposed approach to transform an original mesh without interface elements to another with interfaces in the selected places. Nodes named with sub index L, represent the nodes to interpolate the Lagrange multipliers field. Nodes with sub index 0 are nodes corresponding to the original mesh meanwhile the rest, are duplicated nodes to interpolate displacement field.

The Algorithm 2 shows the general structure of the method. The input data are the node coordinates of a standard mesh, a connectivity table (the last column correspond to the material number) and the places where the interfaces are needed (see Figure 7 (a) for an exploded mesh in a certain material and (b) interface between two different materials). The output data are the coordinates of the nodes used to interpolate the displacements, the coordinates of the nodes used for Lagrange multipliers (not needed, just for control), the table of connectivities for bulk elements and the table of connectivities for interfaces elements. 


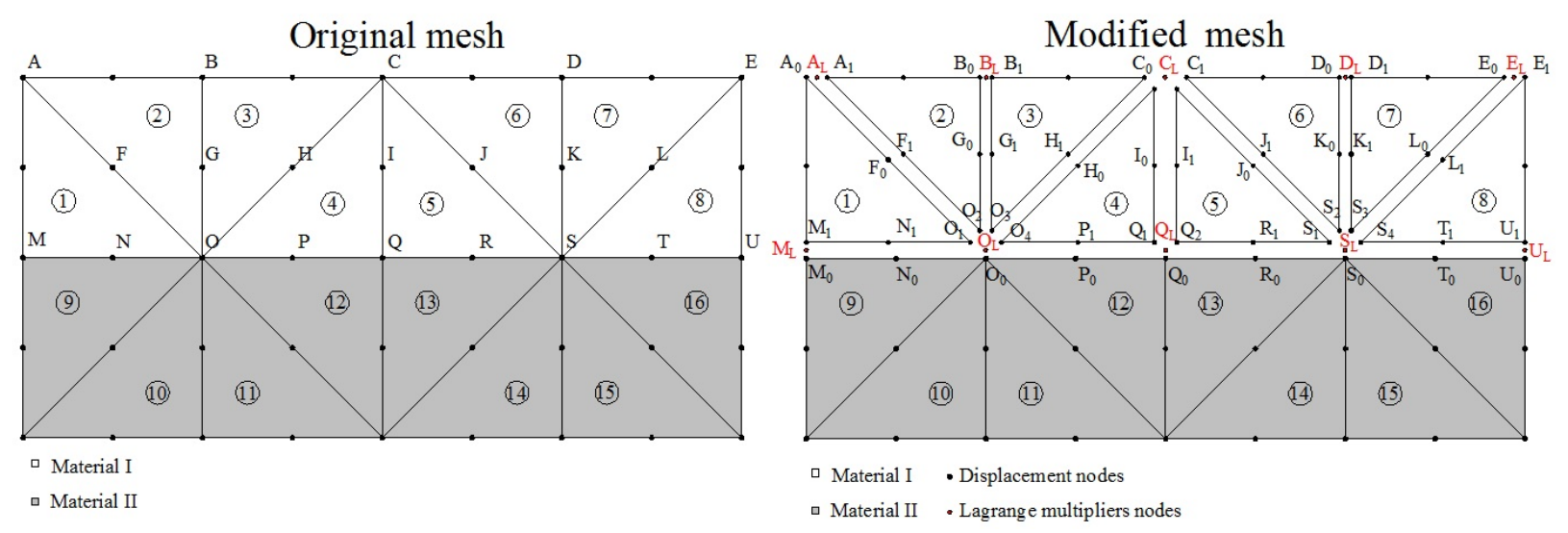

(a) Insertion of interface elements between bulk elements of a certain material.

Original mesh

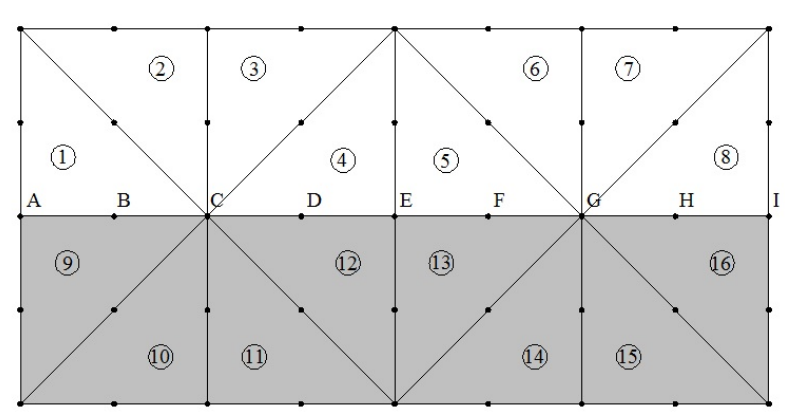

$\square$ Material I

口 Material II
Modified mesh

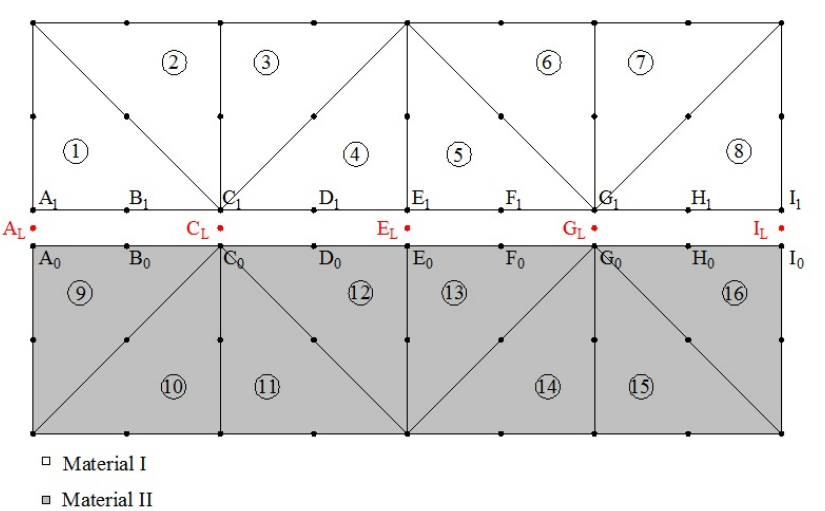

(b) Insertion of interface elements between different materials.

Figure 7. Different approaches to insert interface elements.

The general algorithm is splitted in three stages: the first stage, detailed in Algorithm 3, looks for the nodes in the original mesh which needs to be duplicated. In this algorithm, the concept of support is used. The support of a node $I$ represents all the elements that share the node $I$. For example in figure 7 (a), the support of node $Q$ is $\left[\begin{array}{llll}4 & 5 & 12 & 13\end{array}\right]$. This matrix is crucial for the definition of the interfaces. The criteria used to decide whether a node needs to be duplicated or not, depends on the place where the interface is required. When the interface is required between bulk elements of the same material number $n$, the node $I$ to be duplicated is the one that has at least one element with material $n$ in its support. When the interfaces are required between different materials, the number of different materials in the support must be bigger than one. This stage finishes with all node numbers to be duplicated and with the respective support.

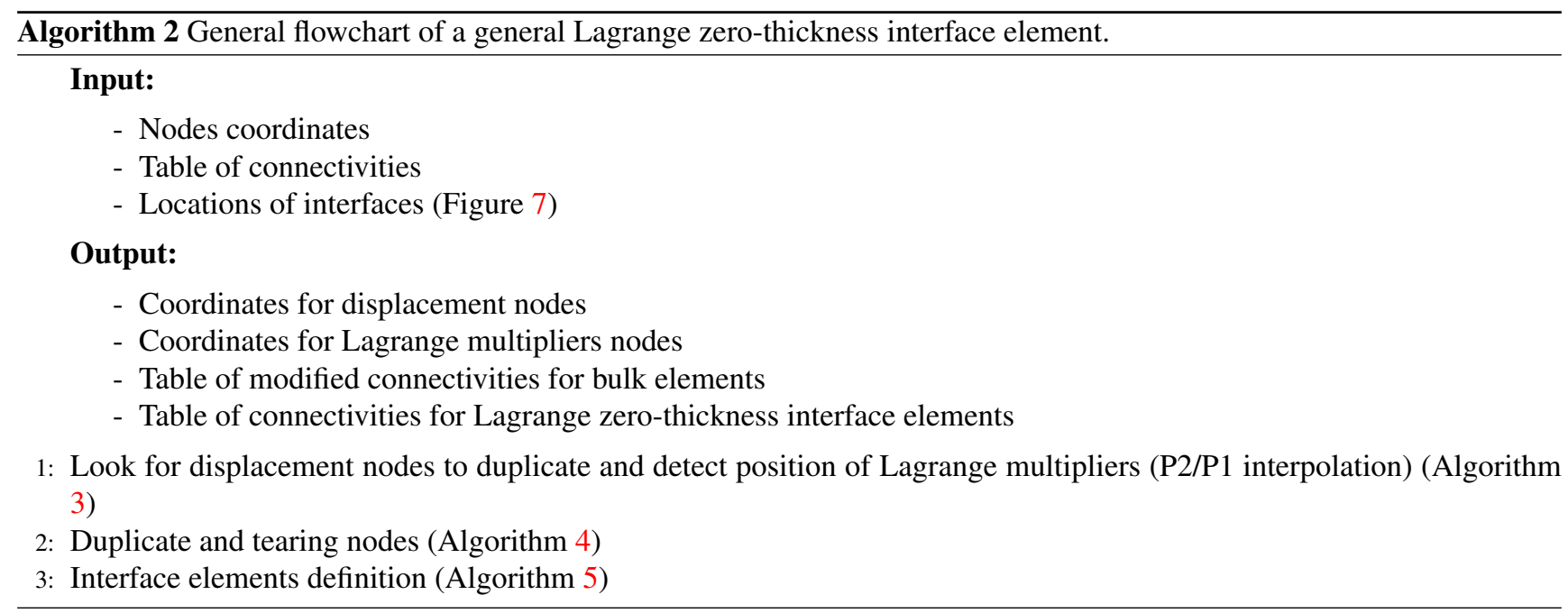


The second stage is summarized in Algorithm 4 and consists of an strategy to duplicate the corresponding nodes and modify the original connectivity table (tearing nodes). The third stage is detailed in Algorithm 5. The interface elements are defined in this algorithm following the numeration exposed in Figure 7 for both cases: (a) interfaces belonging to sides of the same material (interface number 1) and (b) interfaces between different materials (interface number 2).
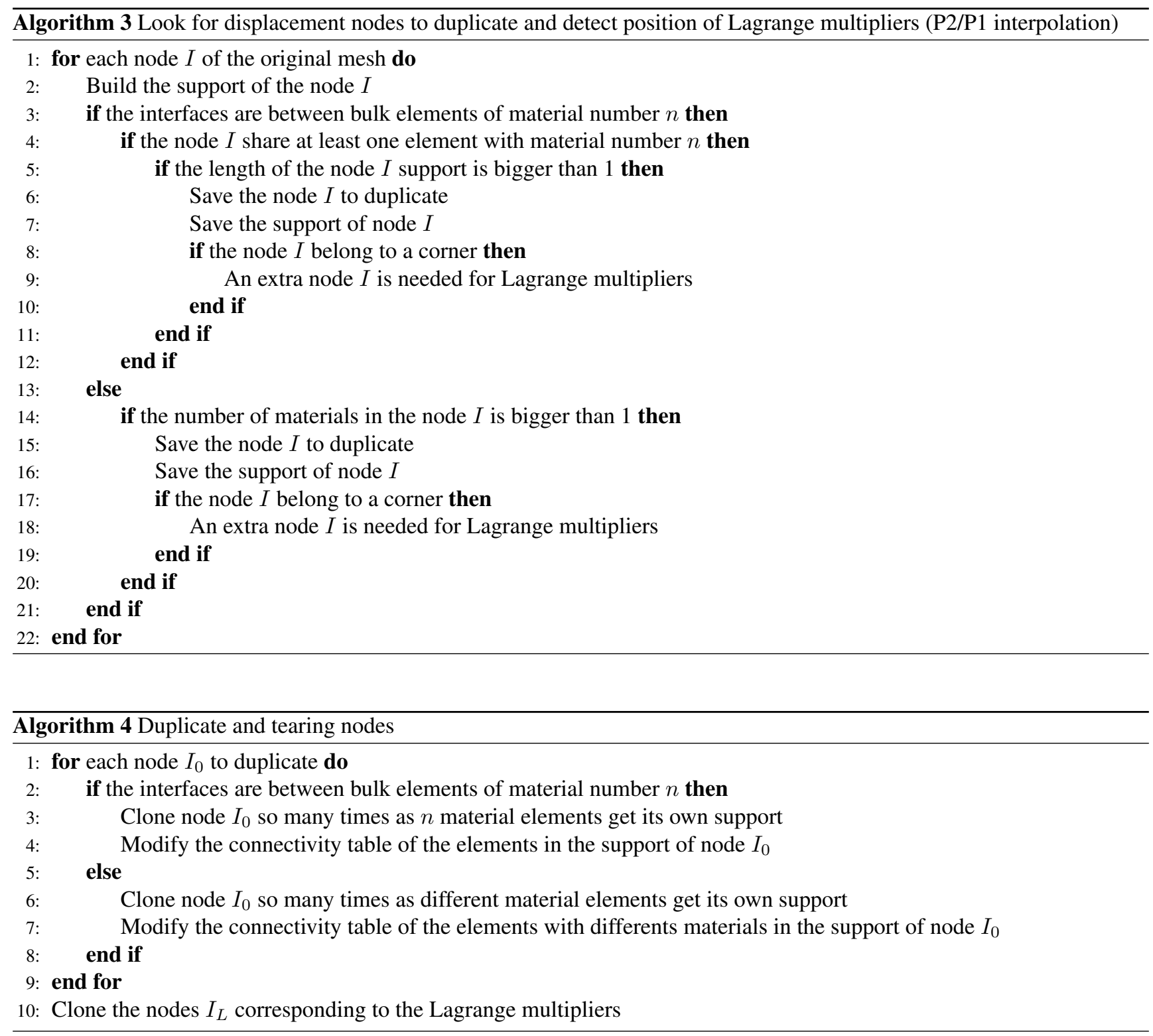

Remark 2. Two different approaches to locate the Lagrange multiplier nodes are showed in Figure 8. As an example, a simple mesh to be exploded is exposed in Figure $8(a)$. In the first approach, a node is assigned for each concurrent interface element to solve the Lagrange multipliers (independent Lagrangian nodes Figure $8(b)$ ), while the second approach uses a single node for the overall concurrent fracture domain (dependent Lagrangian nodes Figure 8 (c)). Dependent Lagrangian nodes are used for plane fractures (delamination, contact), but they become numerically unstable during the softening regime in the case of complex fracture paths (Fiber debonding, matrix cracking), being the independent Lagrangian nodes more appropriated.

Remark 3. Dependent Lagrangian nodes correspond to the discrete space presented in equation (27). Independent Lagrangian nodes does not ensure the continuity in the field, so the discrete space for this case is $\mathscr{X}_{h}=\mathcal{P}_{d}^{1}\left(\Gamma_{h}\right)$.

\section{NUMERICAL EXAMPLES}

\section{Analysis of the effect of the penalty parameter on the solution}

An analysis is carried out to check the well-posedness of the numerical method to deal with a decoupling of the penalty term presented in equation (78). As was discussed in Taylor (1986), the consistency and stability, that is, the uniqueness of the approximate solution must be checked. The following error definitions are used: 


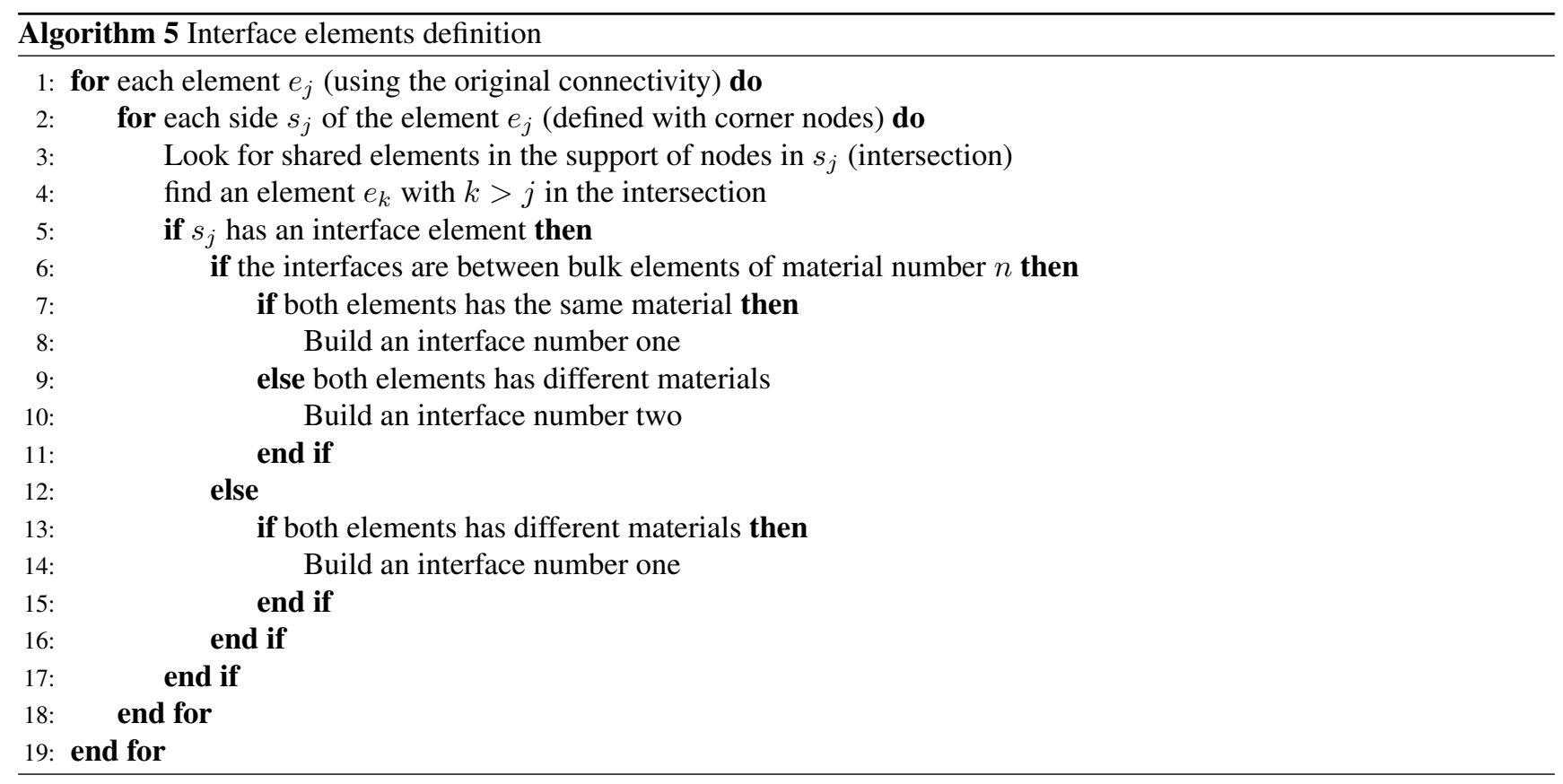

- Displacement nodes

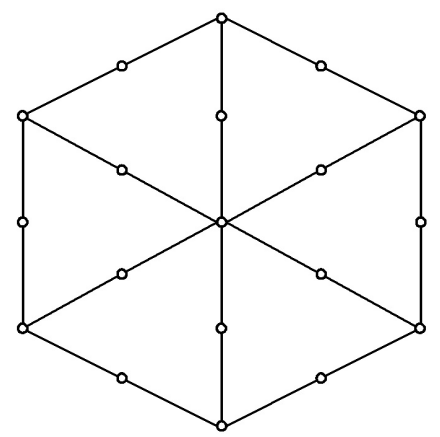

(a) Original Mesh.
- Lagrange multiplier nodes

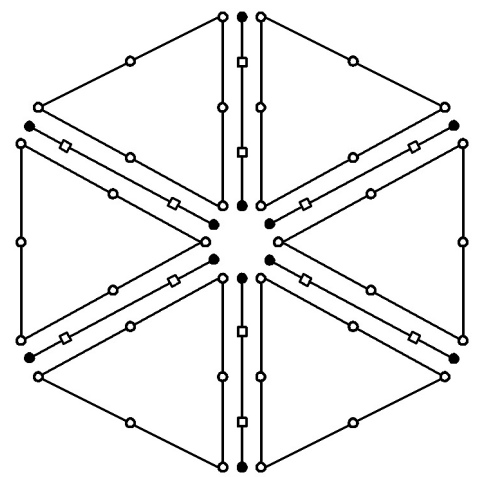

(b) Mesh with independent Lagrangian (c) Mesh with dependent Lagrangian nodes.

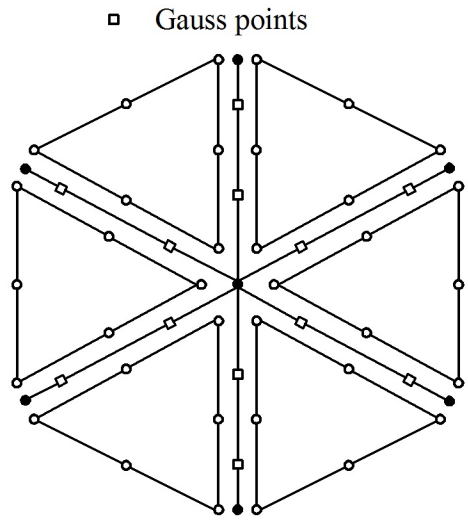

nodes.

Figure 8. Different approaches to insert the Lagrange multiplier nodes.

$$
e^{h}=\frac{\left\|\left\{\mathscr{G}_{3}^{h+1}\right\}-\left\{\mathscr{G}_{3}^{h}\right\}\right\|_{L^{2}(\Gamma)}}{\left\|\left\{\mathscr{G}_{3}^{h}\right\}\right\|_{L^{2}(\Gamma)}} \quad e^{\gamma}=\frac{\left\|\left\{\mathscr{G}_{3}^{\gamma+1}\right\}-\left\{\mathscr{G}_{3}^{\gamma}\right\}\right\|_{L^{2}(\Gamma)}}{\left\|\left\{\mathscr{G}_{3}^{\gamma}\right\}\right\|_{L^{2}(\Gamma)}},
$$

where $\mathscr{G}_{3}=\frac{1}{2}\left[\int_{\partial^{t} \Omega}[\eta \cdot \dot{\widehat{p}}-\dot{\eta} \cdot \widehat{p}] d \partial^{t} \Omega+\int_{\Gamma}[\dot{\hat{\boldsymbol{\lambda}}} \cdot \llbracket \boldsymbol{u} \rrbracket+\widehat{\boldsymbol{\lambda}} \cdot \llbracket \dot{\boldsymbol{u}} \rrbracket] d \Gamma\right]$ is the released energy, with $\widehat{\boldsymbol{\lambda}}=\boldsymbol{\lambda}+\gamma(\llbracket \boldsymbol{u} \rrbracket-\boldsymbol{\delta})$ and $\widehat{p}(x, t)=\overline{\boldsymbol{p}}(x) \cdot \boldsymbol{u}(x, t)$.

The released energy is a scalar that summarizes all variables interacting in the fracture, and the vector $\left\{\mathscr{G}_{3}^{\bullet}\right\}$ used in equations (77), contains this quantity computed per each load step. The error $e^{h}$ measures the evolution of $\mathscr{G}_{3}$ using a mesh $h+1$ respect to the previous coarser mesh $h$, and $\boldsymbol{e}^{\gamma}$ the evolution with a penalty increment from $\gamma$ to $\gamma+1$. The following definition for $\gamma$ is adopted for the case $\beta=2$ in equation (49):

$$
\gamma^{j}>\max _{0<\delta_{e q}<\delta_{c}}\left|\psi^{\prime \prime}\left(\delta_{e q}\right)\right| \Rightarrow \gamma^{j}=\alpha \frac{\sigma_{c}^{j}}{\delta_{c}^{j}}, \text { with } \alpha>1,
$$

where the superscript $j$ represents the material number $(j=1,2)$, and the parameter $\alpha$ is the same for different penalties and belong to the arbitrary set $\alpha=\{5,10,50,100,500,1000,5000,10000\}$. The discussion about the penalty parameter for the case of different values of $\beta$ is beyond the scope of this paper and will be addressed in future work.

Figure 9 shows the structure proposed for the patch test. The elements shown in Figure 3 are used for the different discretizations that are detailed in Table 1, where dependent Lagrangian nodes are used. Different values for the fracture parameters presented in Table 2 are assumed for the interfaces between the bodies to study the evolution of the numerical solution. 


\begin{tabular}{c|c|c|c|c} 
& Interface length $[\mathrm{mm}]$ & $\mathrm{N}^{\mathrm{o}}$ of T6 elements & $\mathrm{N}^{\circ}$ of Q8 elements & $\mathrm{N}^{\mathrm{o}}$ of interfaces \\
\hline Mesh 1 & 5 & 16 & 4 & 2 \\
\hline Mesh 2 & 1 & 200 & 100 & 10 \\
\hline Mesh 3 & 0.2 & 5000 & 2500 & 50 \\
\hline Mesh 4 & 0.1 & 20000 & 10000 & 100 \\
\hline Mesh 5 & 0.0666667 & 45000 & 22500 & 150
\end{tabular}

Table 1. Mesh properties used in the patch test.

\begin{tabular}{c|c|c|c} 
& Bulk Prop. & Interface mat. $\mathrm{N}^{\mathrm{o}} 1$ & Interface mat. $\mathrm{N}^{\mathrm{o}} 2$ \\
\hline Young modulus E $[\mathrm{MPa}]$ & 100000 & - & - \\
\hline Poisson modulus & 0.25 & - & - \\
\hline Fracture stress initiation $\sigma_{c}[\mathrm{MPa}]$ & - & 50 & 25 \\
\hline Fracture energy $G_{c}[\mathrm{~N} / \mathrm{mm}]$ & - & 0.5 & 0.5
\end{tabular}

Table 2. Material properties used in the patch test.

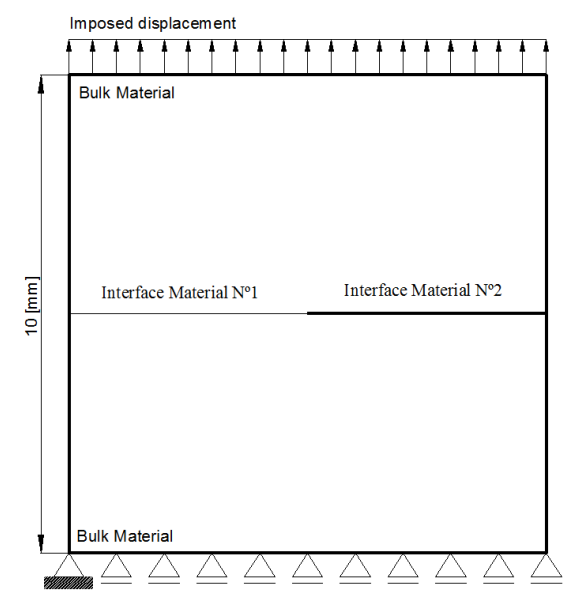

(a) Tension patch test.

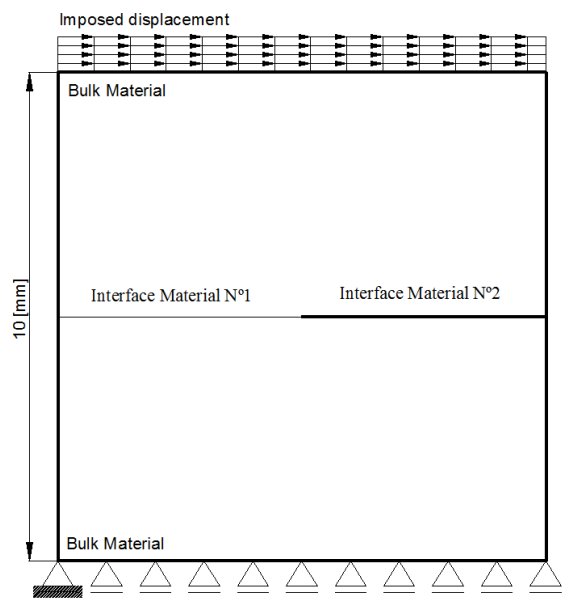

(b) Shear patch test.

Figure 9. Structure used for patch test and force-displacement results.

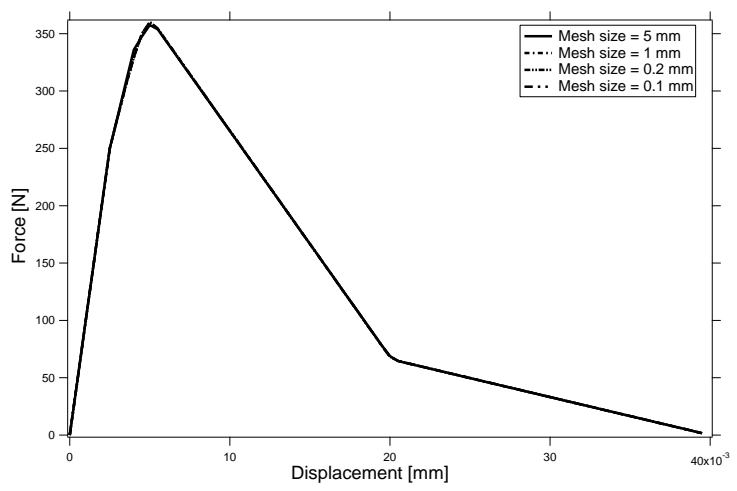

(a) Force versus displacement for tension patch test for $\alpha=10$

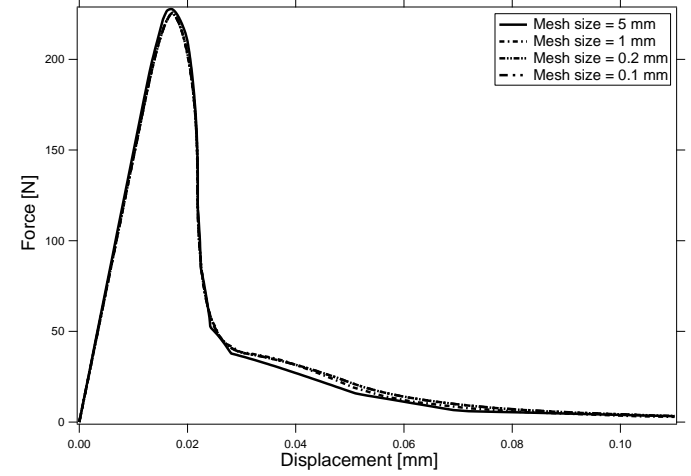

(b) Force versus displacement for shear patch test for $\alpha=$ 100.

Figure 10. Mechanical response of the patch test.

The force at the top of the cell versus the imposed displacement corresponding to $\alpha=100$ for the cases of normal and tangential displacement are plotted in Figure 10 (a) and (b) showing the effect of the mixture of different interface properties for tension and shear loads. The evolution of error measure $e^{h}$ as a function of mesh refinement is plotted in Figure 11(a), showing that the proposed decoupling of the penalty parameter maintains the consistency of the results. The Figure 11(b) shows that if the parameter $\alpha$ increases, the relative error $e^{\gamma}$ is reduced but not monotonically. See for example the results 

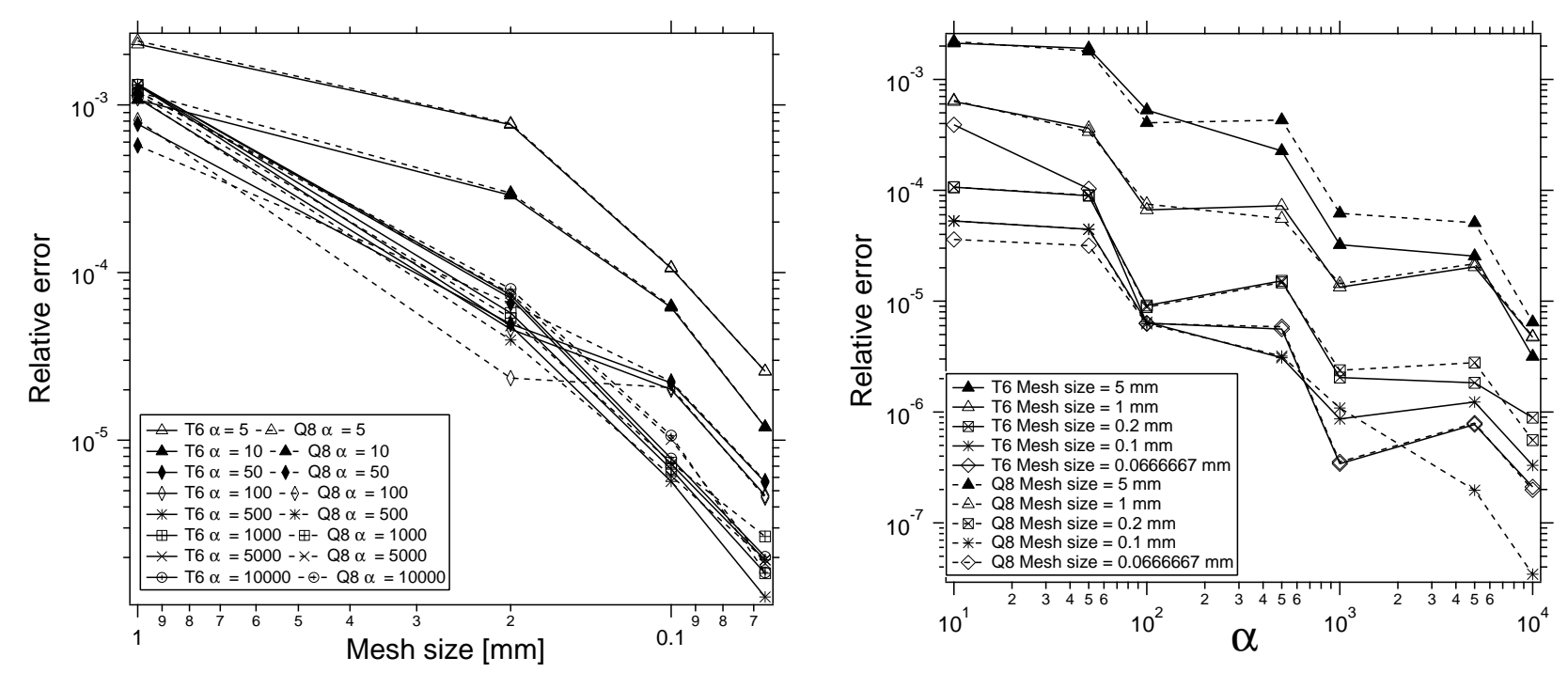

(a) Computed relative error $\boldsymbol{e}^{h}$ as a function of the mesh size for Q8/L2 and (b) Computed relative error $\boldsymbol{e}^{\gamma}$ as a function of the $\alpha$ parameter for Q8/L2 T6/L2 interpolations. and $\mathrm{T} 6 / \mathrm{L} 2$ interpolations.

Figure 11. Relative error evolution.

corresponding to meshes 1,3 and 5 for the case $\alpha=500$. For values of $\alpha$ higher than the exposed in Figure 11 (b), the model does not always converge, so from now on $\alpha \in[5,10000]$ is used. Previous results suggest that the use of selective penalty parameters in terms of the material properties is an accurate approach for the following examples when the interpolation exposed in sets (26) and (27) is used.

\section{Matrix fibre debonding and matrix cracking.}

This example represents the fracture crack path of a square cell fibre reinforced matrix subjected to an uni-axial displacement. The geometry of the problem is shown in Figure 12 (a), material properties for the fibres, matrix and interface are given in Table 3, the used meshes are presented in Table 4, and a mesh detail is presented in Figure 12 (b). Six-nodes triangles and independent Lagrangian nodes are implemented in both examples presented in this section.

\begin{tabular}{c|c|c|c} 
& Matrix & Fibre & Interface material \\
\hline Young modulus E [MPa] & 4000 & 40000 & - \\
\hline Poisson modulus & 0.4 & 0.33 & - \\
\hline Fracture stress initiation $\sigma_{c}[\mathrm{MPa}]$ & 30 & - & 10 \\
\hline Fracture energy $G_{c}[\mathrm{~N} / \mathrm{mm}]$ & 0.25 & - & 0.05
\end{tabular}

Table 3. Material properties for fibre reinforced matrix.

Figure 12 (c) shows the total reaction force in the top of the cell versus the impose displacement. These results show a good agreement with the results presented by Nguyen (2014) despite the use of totally different meshes (Table 4). The post peak stage has a better concordance for Mesh 1 because the mesh size used in Nguyen (2014) is similar to mesh 1 near the edge of the cell.

\begin{tabular}{c|c|c|c|c|c} 
& $\mathrm{N}^{\mathrm{o}}$ Bulk elem. & $\mathrm{N}^{\mathrm{o}}$ interf. elem. & $\mathrm{N}^{\mathrm{o}}$ displ. nodes & $\mathrm{N}^{\mathrm{o}}$ Lagrange mult. & $\alpha$ parameter \\
\hline Mesh in Nguyen (2014) & 7014 & 5080 & 12014 & - & - \\
\hline Mesh 1 & 706 & 812 & 3643 & 322 & 1000 \\
\hline Mesh 2 & 9210 & 10562 & 46828 & 3755 & 1000
\end{tabular}

Table 4. Mesh properties for the fiber reinforced matrix.

All the material degradation stages presented in experimental results in París et al. (2007) can be identified in the results plotted in Figure 13 (a). The snapshot A shows the debonding phenomena prior to the matrix cracking. Snapshot B shows with circles the kinking of the crack. The arrested fracture in the superior part and the unstable propagation in the downward part of the cell, are presented in snapshot C. The final rupture stage is presented in snapshot D. Figure 13 (b) shows the experimental results for transverse cracking in a fibrous composite material París et al. (2007). 


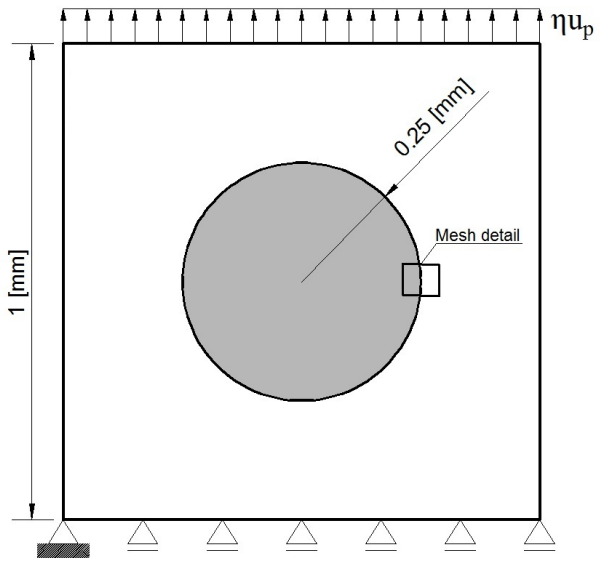

(a) Proposed problem.

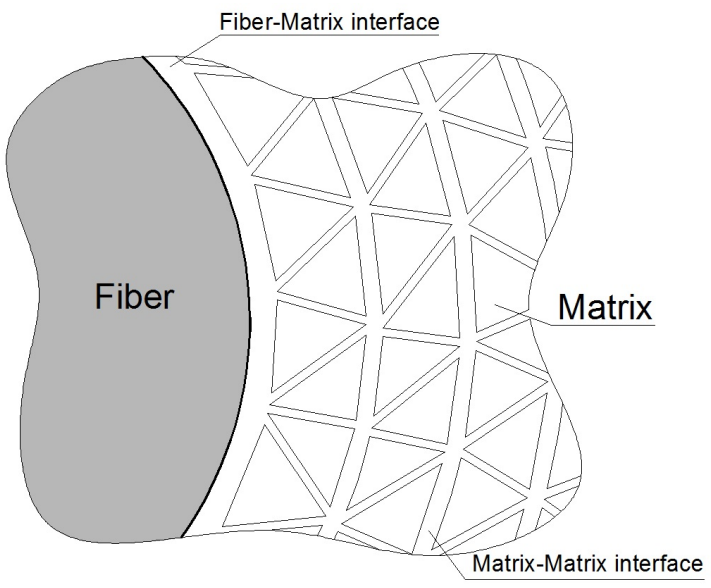

(b) Detail of the used mesh on Figure 12 (a).

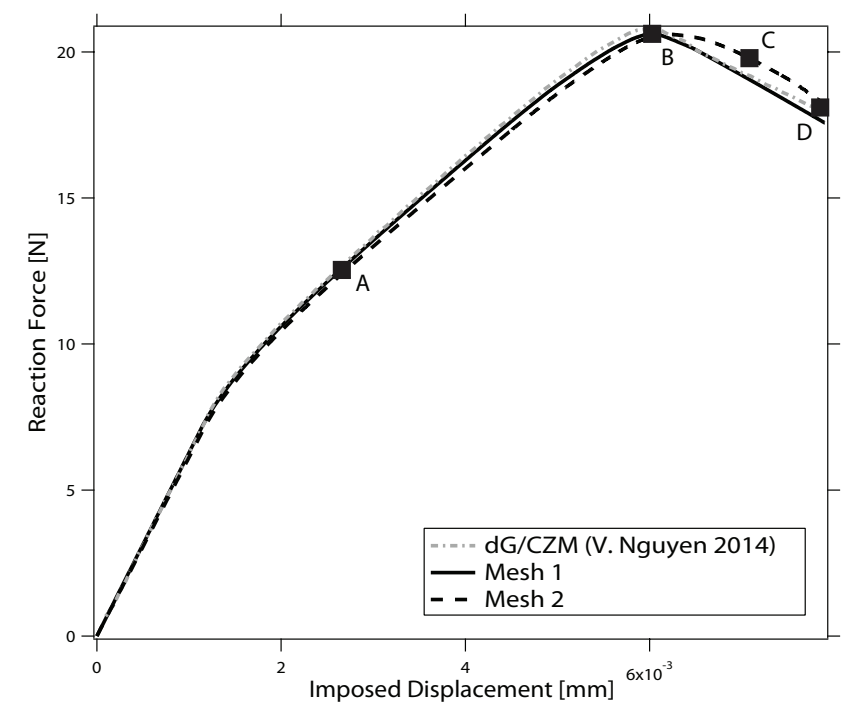

(c) Comparison between the proposed model and a discontinuous Galerkin formulation proposed in Nguyen (2014).

Figure 12. Proposed problem for transverse fracture of fiber matrix composite.

Following the concept of the last example, an extended example using a bigger cell of a composite material is proposed for transverse cracking analysis. A deeper analysis of the mesh sensitivity of the method is carried out and the results are compared with those obtained by Cid Alfaro et al. (2010). Figure 14 shows the simulated square cell with $125 \mu \mathrm{m}$ side and a $30 \%$ of volumetric fiber content. Table 5 shows the mechanical properties used for the matrix and the fibers, and Table 6 presents the meshes used by Cid Alfaro et al. (2010) and those used in this paper.

\begin{tabular}{c|c|c|c} 
& Matrix & Fiber & Matrix-Fiber \\
\hline Young Modulus E $[\mathrm{MPa}]$ & 3900 & 86900 & - \\
\hline Poisson Modulus & 0.37 & 0.23 & - \\
\hline Initiation stress $\sigma_{c}[\mathrm{MPa}]$ & 50 & - & 25 \\
\hline Fracture energy $G_{c}[\mathrm{~N} / \mathrm{mm}]$ & 0.5 & - & 0.5
\end{tabular}

Table 5. Material properties for longitudinal fiber reinforced epoxy matrix.

The crack pattern obtained with the proposed model is presented in Figures 15. The results show a low dependence on the mesh density, and the failure mechanism obtained is conceptually similar to that of the single-fiber example. Failure starts with a debonding followed by a crack propagation within the matrix, first in a diffuse sense and finally with crack nucleation.

The obtained numerical results are consistent with the experimental results observed in Figure 13 (b) (París et al. 2007) and Figure 14 (b) and (c) (París et al. 2007; Hobbiebrunken et al. 2006). The crack is first propagated within the matrix, then follows an arc-length of each fibre interface corresponding to a central angle of approximately $60^{\circ}$ (Figure 13 (b)). Figure 16 shows the load-displacement curves obtained and the comparison with the solution in Cid Alfaro et al. (2010). 

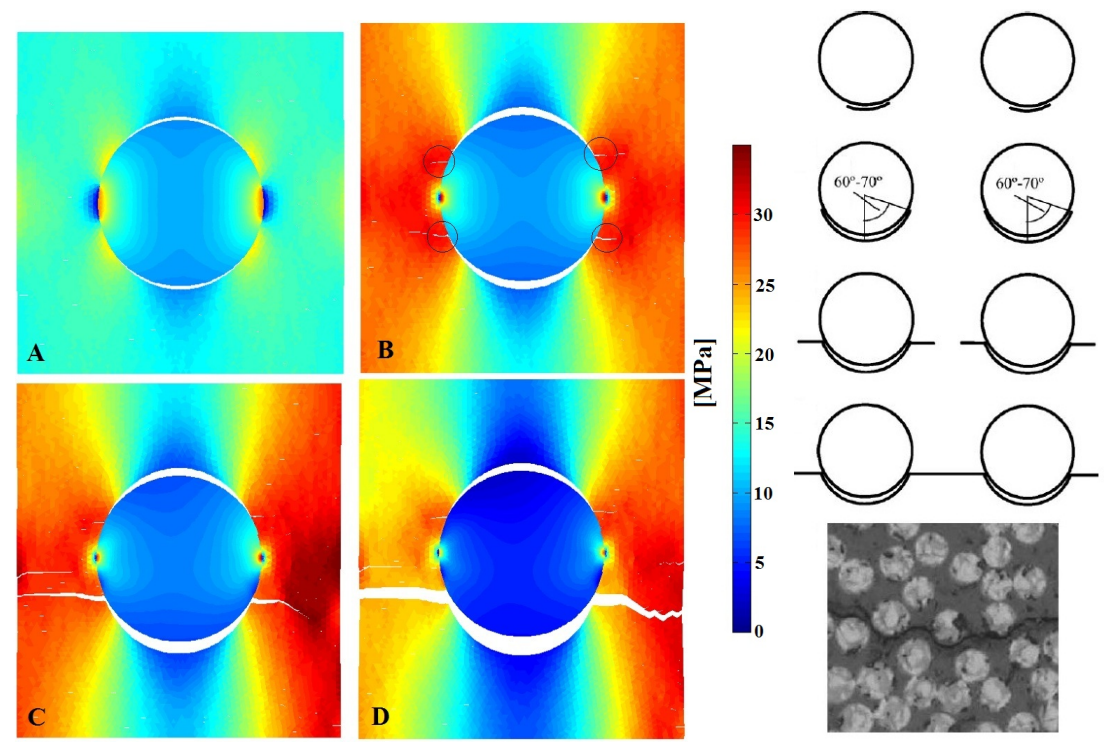

(a) Numerical result of matrix fiber debonding with matrix cracking (b) Experimental results prefor Mesh 2. Displacements increased by ten. sented in París et al. (2007).

Figure 13. Comparison with experimental results.

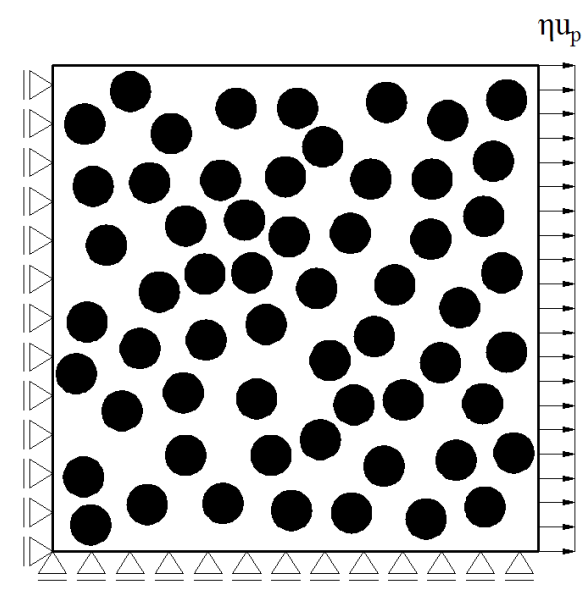

(a) Proposed cell geometry.

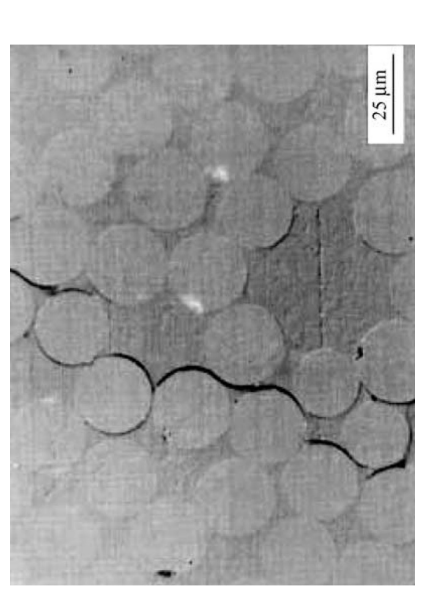

(b) Experimental results presented (c) Experimental results presented by Hobbiebrunken et al. by París et al. (2007).
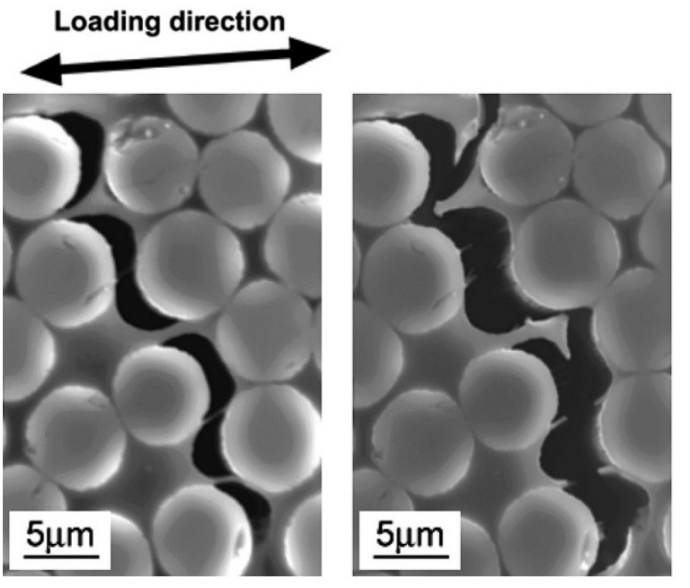

(2006).

Figure 14. Transverse fracture of an epoxy matrix reinforced with longitudinal fibers.

\begin{tabular}{c|c|c|c|c|c} 
& $\mathrm{N}^{\mathrm{o}}$ Vol. Elem. & $\mathrm{N}^{\mathrm{o}}$ Interfaces & $\mathrm{N}^{\mathrm{o}}$ Displ. Nodes & $\mathrm{N}^{\mathrm{o}}$ Lagrang. Nodes & $\alpha$ parameter \\
\hline Cid Alfaro et al. (2010) & 9566 & 10454 & - & - & - \\
\hline Mesh 1 & 2949 & 3310 & 14679 & 6620 & 100 \\
\hline Mesh 2 & 14662 & 16045 & 72219 & 32089 & 4000 \\
\hline Mesh 3 & 19640 & 21251 & 96128 & 42501 & 10
\end{tabular}

Table 6. Mesh properties for longitudinal fiber reinforced epoxy matrix.

The adimensional displacement is calculated as the imposed displacement divided by the ultimate displacement of the matrix $\boldsymbol{u}_{p} / \delta_{c}^{m}$, while the adimensional traction is computed as the traction reaction in the cell edge divided by the critical stress of the matrix $\sigma_{c}^{m}$. Despite the differences in the geometrical position of the fibers, and the difference of the meshes used in this paper with those used by Cid Alfaro et al. (2010), the load-displacement curves are similar even for the case of the coarsest mesh (Mesh 1). 

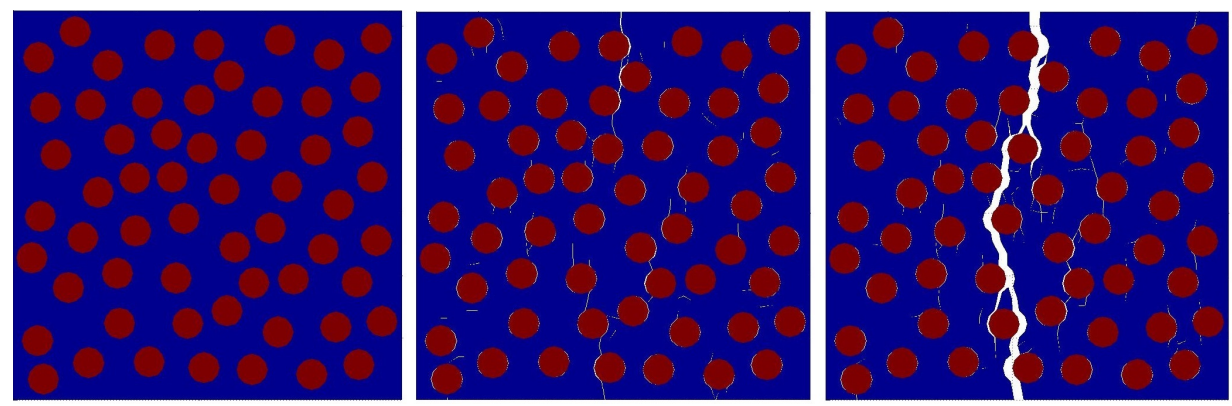

(a) Transverse fracture in a longitudinal fiber reinforced epoxy composite for Mesh 1.
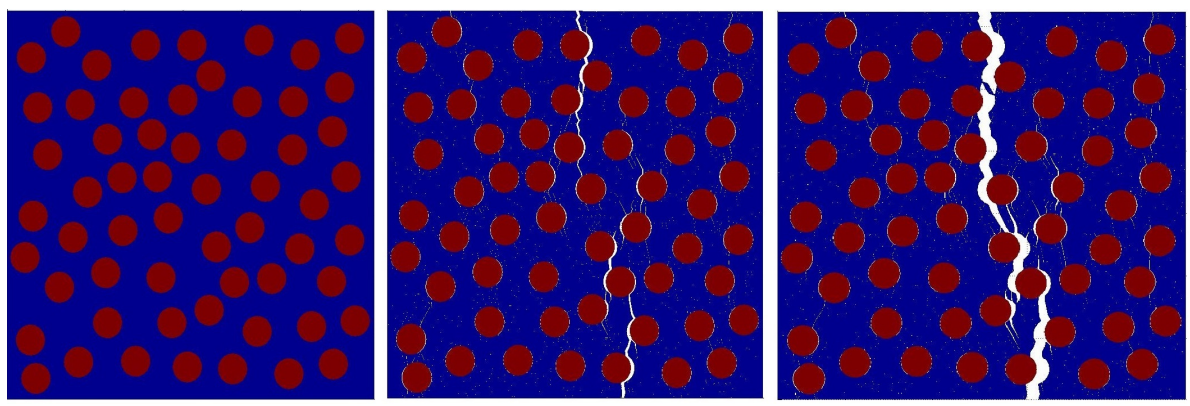

(b) Transverse fracture in a longitudinal fiber reinforced epoxy composite for Mesh 2.
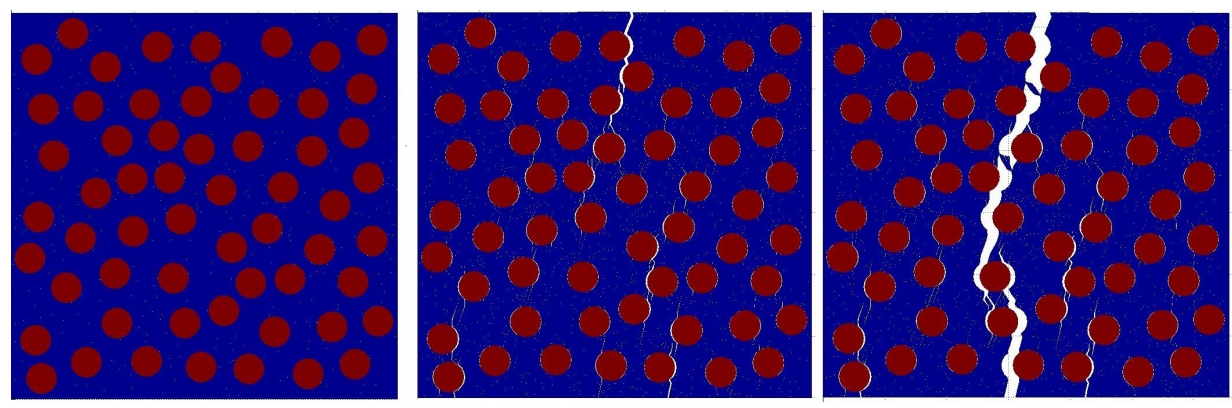

(c) Transverse fracture in a longitudinal fiber reinforced epoxy composite for Mesh 3.

Figure 15. Transverse fracture in a longitudinal fiber reinforced epoxy composite for Mesh 1.

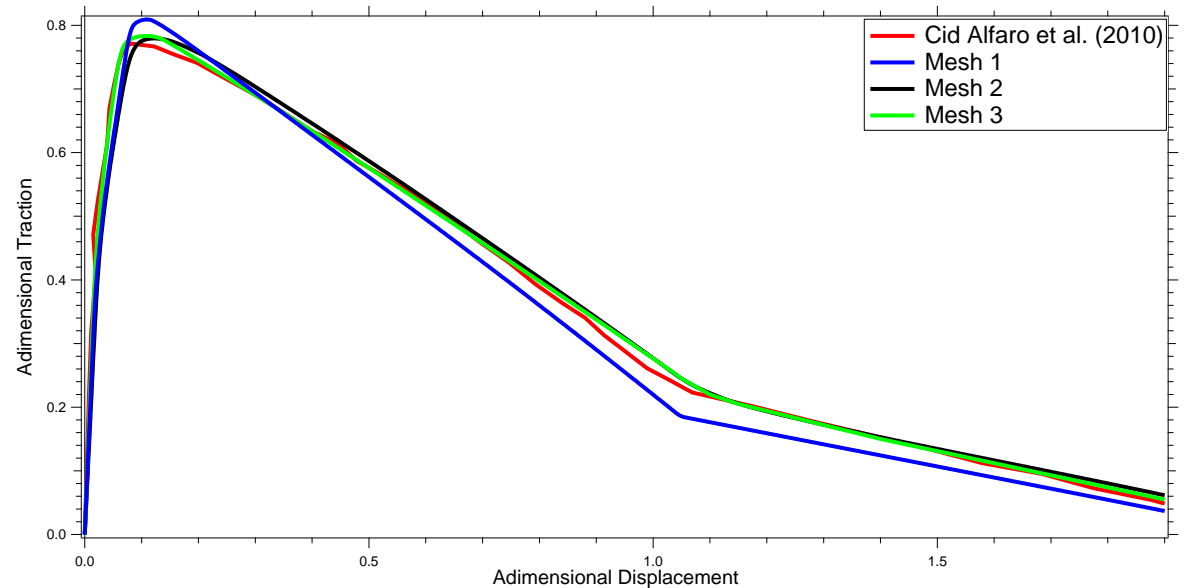

Figure 16. Adimensional traction versus adimensional displacement.

\section{Meso-mechanical simulation of a L-shape concrete specimen.}

The proposed formulation is used to model a L-shape concrete specimen tested by Winkler et al. (2001). The meso-structure is taken into account with a representative cell embedded in zone of interest of the macro-structure. Three phases are considered in these cells: a cementitious matrix, aggregate particles and matrix/aggregate interfaces. The material properties 
corresponds to a low-moderate resistance concrete, giving a crack mechanism analogously to that presented in the last example, starting with matrix/particle debonding followed by fracture propagation within the cementitious matrix. The following conditions are hypothesis considered for the proposed model: plane stress state, elastic behavior for the aggregate, matrix-interface critical tension weaker that the critical tension of the cementitious matrix and finally a matrix susceptible to be cracked.

Figure 17(a) presents the geometry of the concrete specimen that is $100 \mathrm{~mm}$ thick. $F$ is the applied force and $\delta$ is the displacement measured in the experiment. Figure 17(b) shows the fracture pattern obtained in the tests (Winkler et al. 2001). The criteria used by Du et al. (2013) is adopted in this paper for the generation of the meso-structure. The aggregate is placed over the striped zone in Figure 17(a) using the Montecarlo Method.

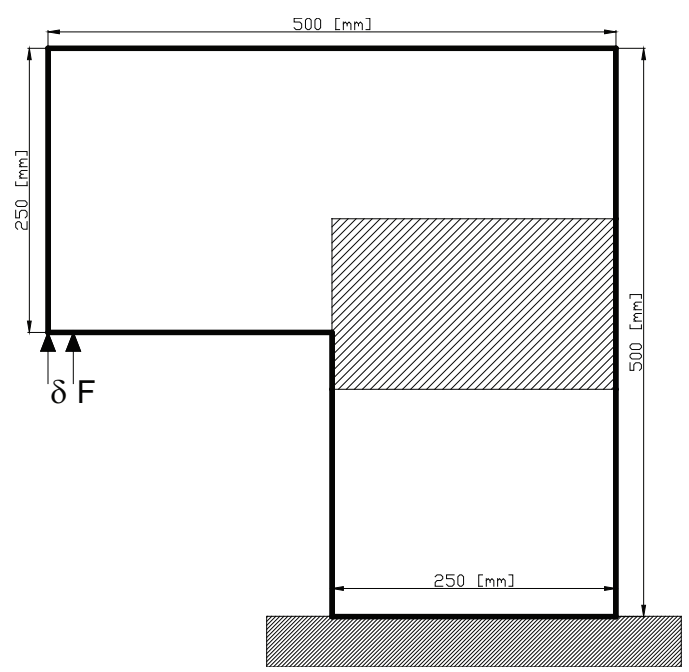

(a) Geometry and applied load to the specimen.

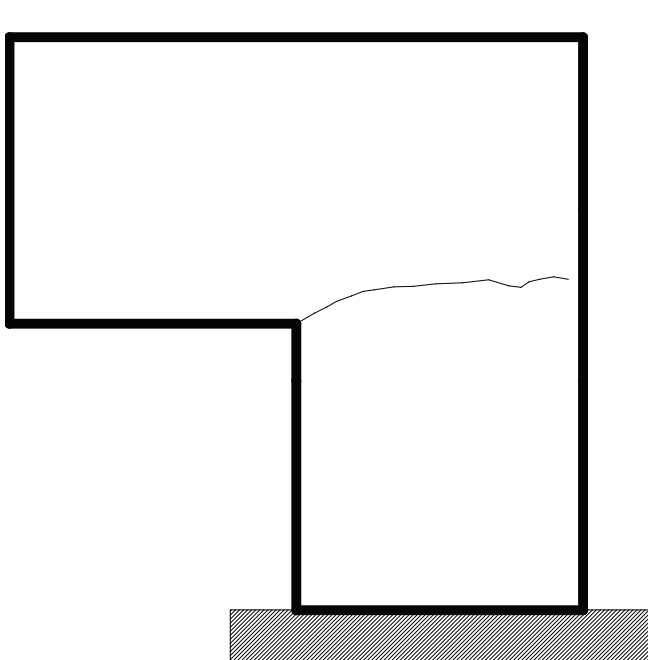

(b) Crack pattern experimentally obtained (Winkler et al. 2001).

Figure 17. Mechanical problem considered for the analysis.

The three realizations presented in Figure 18 were performed for the meso-structure, using 6-nodes triangles and independent Lagrangian nodes for discretization. An aggregate volumetric ratio of $50 \%$ was considered. The material parameters used for each phase are presented in Table 7, and they are in agreement with those adopted by other researchers to model the same problem (Toro et al. 2016; Carol et al. 2001; Unger and Eckardt 2011). For all the examples exposed in this section, the penalty parameter is fixed in $\alpha=10$.

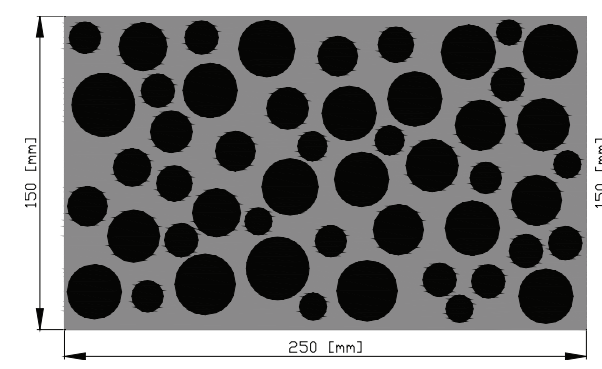

(a) Meso-structure 1.

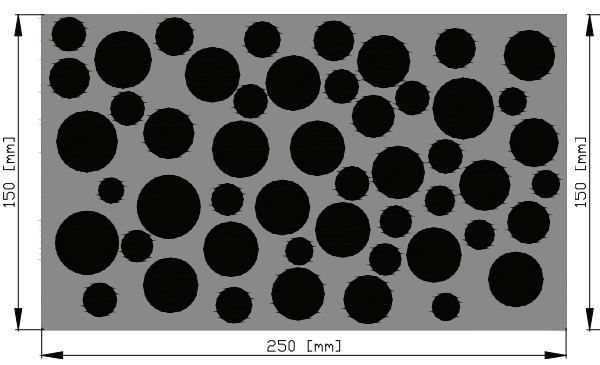

(b) Meso-structure 2.

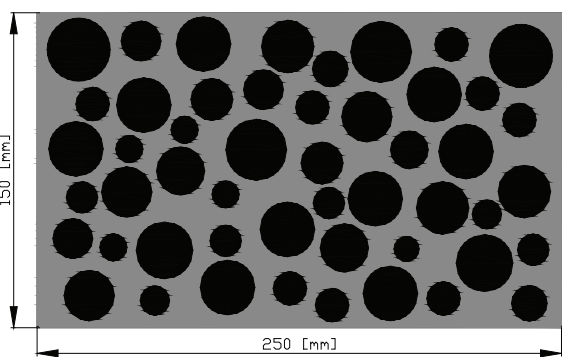

(c) Meso-structure 3.

Figure 18. Meso-structural realizations. Aggregate Volume $50 \%$.

\begin{tabular}{c|c|c|c} 
& Matrix & Aggregate & Matrix-Aggregate \\
\hline Young Modulus E $[\mathrm{MPa}]$ & 18500 & 37000 & - \\
\hline Poisson Modulus & 0.22 & 0.16 & - \\
\hline Critical Stress $\sigma_{c}[\mathrm{MPa}]$ & 2.6 & - & 1.3 \\
\hline Fracture Energy $G_{c}[\mathrm{~N} / \mathrm{mm}]$ & 0.140 & - & 0.070
\end{tabular}

Table 7. Material properties considered in each phase of the concrete. 
Figures 19 show the final crack pattern obtained for each meso-scale configuration. The fracture process starts at the corner of the L-shape specimen, growing through the weaker region of aggregate-mortar interface, linked by crack within the matrix.

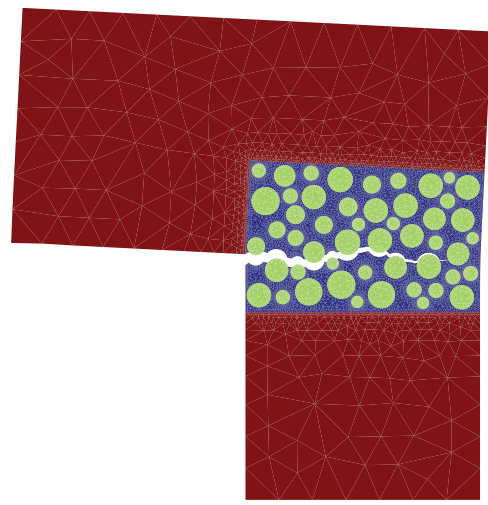

(a) Final configuration for meso- (b) Final mechanical realization 1 . Volume aggregate mechanical $50 \%$. Displacements $x 25$.

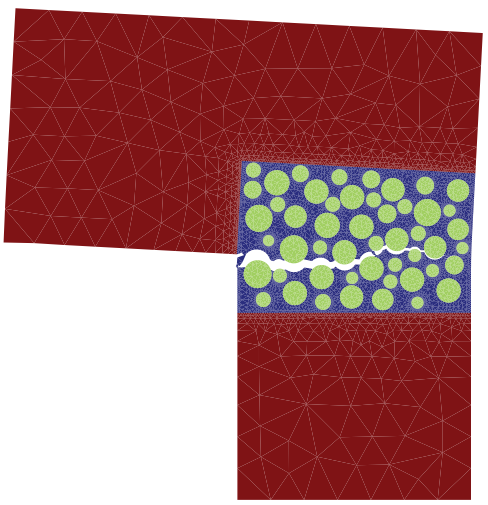

configuration for meso- (c) Final configuration for mesorealization 2. Volume mechanical realization 3. Volume aggregate aggregate $50 \%$. Displacements $x 25$. $\quad 50 \%$. Displacements $x 25$.

Figure 19. Simulated structure. Volume aggregate $50 \%$.

The numerical results obtained with the proposed model are compared with experimental results (Winkler et al. 2001) in Figure 20. The numerical force-displacement curves corresponding to the three proposed realizations are included in Figure 20 (a). Curves are separated in three stages: the elastic regime until about the $50 \%$ of the peak load where no damage is reached; a hardening stage where a crack, normal to the load direction, starts to propagate, and a post-peak or softening regime where the structural member lost its resistance. Unlike fracture mechanism of samples under uni-axial loads where the material undergoes micro-cracks before the final localization (Rodrigues et al. 2016), in this case, the localization is explicitly observed since the beginning of the dissipation process. The model presents a good agreement with experimental results, being the numerical curve among the experimental curves. Figure 20 (b) overlies the fracture pattern obtained in each realization, with the one obtained experimentally(Winkler et al. 2001). Although, in contrast with other approaches (Du et al. 2013), a non-dissipative nature was assumed for the aggregates, the numerical crack path approximates the experimental one in all cases. The assumption of elastic aggregates is proper of a low-strength concrete, for which the interface is much weaker than the aggregates.

Realization 3 plotted in blue, has a better agreement with the experimental results. Realization 2 and 3 plotted with red an black respectively, move away from the experimental pattern showing that fracture phenomena has a random nature with a strong dependence on the micro structural configuration. However, the tendency of the path is clear and the numerical results are reasonably well-posed.

Because of the lack of data about the concrete dosage in the original experimental contribution, an analysis of the influence of coarse aggregate volume ratio in the model is proposed. Using the same approach to generate the meso-structure, a coarse aggregate volume of $20 \%$ showed in Figure 21 is considered. Force-displacement curves and the obtained crack patterns are plotted in Figure 22.

It is observed in Figure 22 (a) that the maximum force obtained in the simulation for this set of realizations is $8[K N]$ approximately, which represents an improvement of $6 \%$ compared with the experimental maximum force. It is clear that, despite the decreasement of a $30 \%$ in the coarse aggregate volume, the variation in the results is relatively low. Due to the lowest resistance of mortar-aggregate interface, the strength increase is related to the decrease of the interface total surface. The post-peak behavior is quite similar compared with the experimental results. Figure 22 (b) shows that the fracture pattern obtained has a similar distribution compared with the case of Figure 20 (b), and the tendency has a clear dependence on the aggregate arrangement.

Two extra examples showed in Figure 23 are considered corresponding to a coarse aggregate volume of $60 \%$. The crack patterns numerically obtained in each case are plotted in Figure 24 while the comparison of load displacement curve and crack paterns numerically obtained with experimental results are presented in Figure 25. A good agreement between the numerical and experimental results is reached for both, peak load value and post-peak behaviour. It can be observed that the numerical crack pattern presents a good agreement with the crack pattern obtained in the tests in the case of realization 2 but not in the case of realization 1.

The proposed examples suggest that the force-displacement curve has low sensitivity to the coarse aggregate volume content. The peak value is more affected by the coarse aggregate content than the post-peak behavior due to the percolation of interfaces (Landis and Bolander 2009). An improvement in the peak load value for lowest aggregate content, and no great change between $50 \%$ and $60 \%$ are observed, while the post-peak behavior is similar for all cases. Similar effects were observed for concrete by other researchers (Rodrigues et al. 2016; López et al. 2008). In contrast, the crack pattern depends 


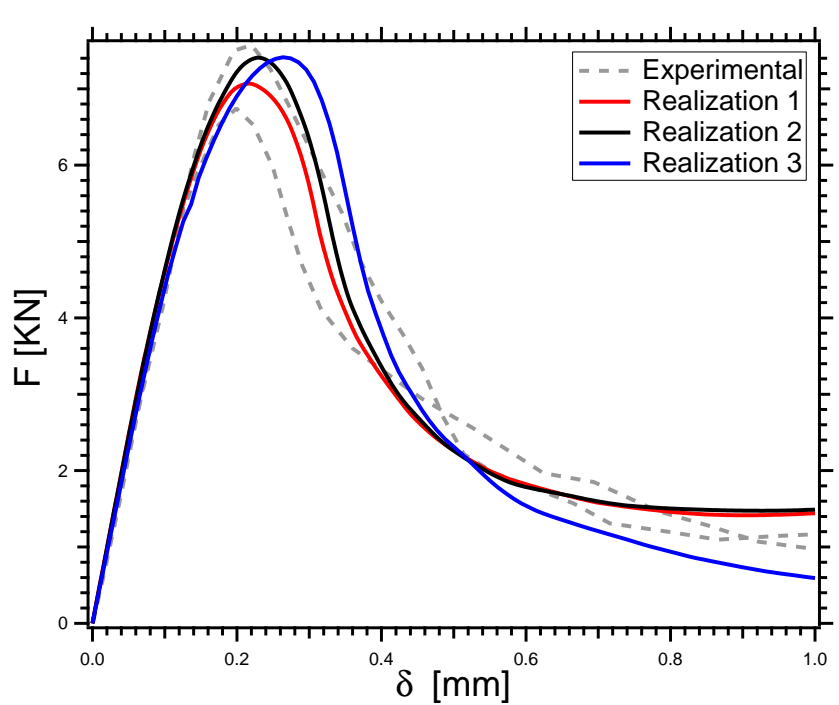

(a) Force versus displacement curves compared with experimental results.

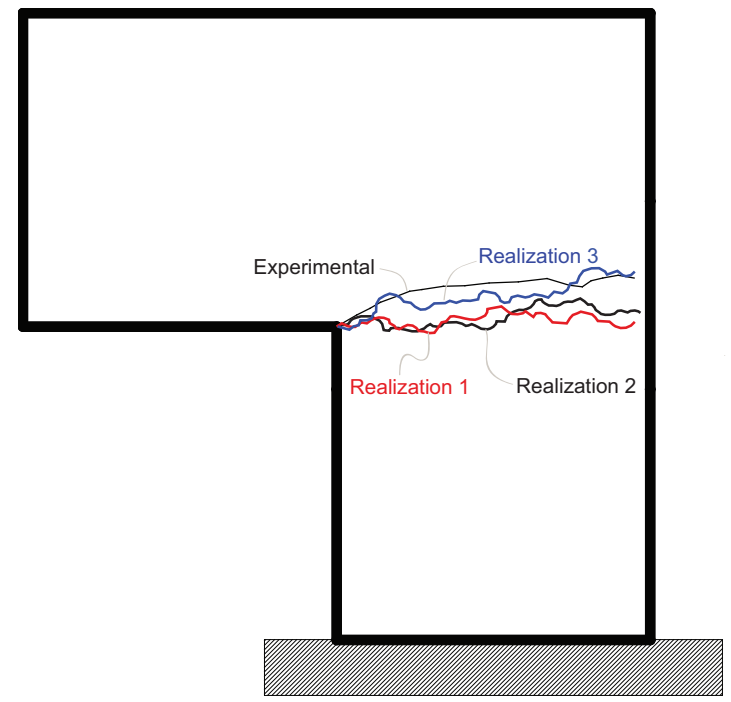

(b) Comparison between the crack pattern numerically and experimentally obtained.

Figure 20. Numerical results computed with the proposed model compared with the experimental results published by Winkler et al. (2001). Coarse Aggregate volume $50 \%$.

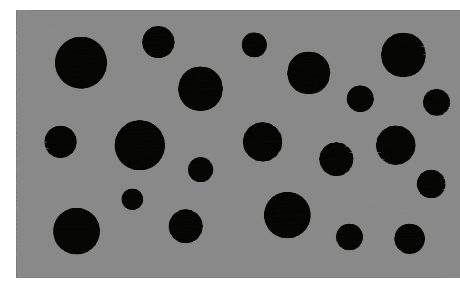

(a) Meso-structure 1.

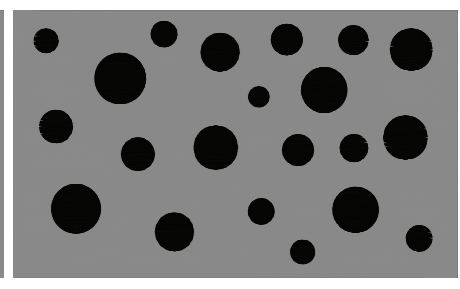

(b) Meso-structure 2.

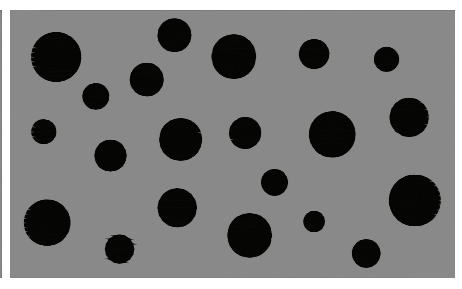

(c) Meso-structure 3.

Figure 21. Meso-structural realizations. Aggregate Volume $20 \%$.

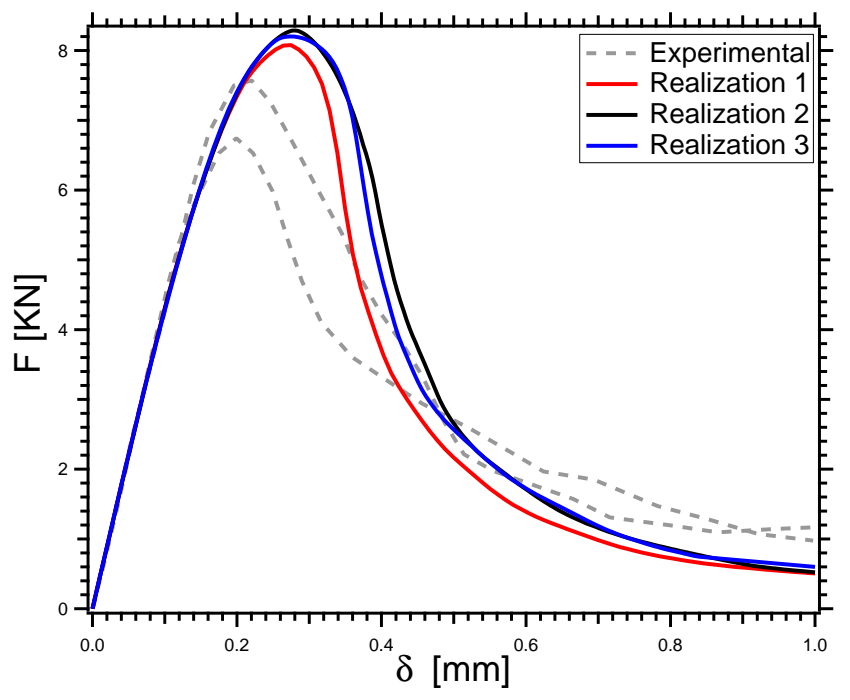

(a) Force versus displacement curves compared with experimental results.

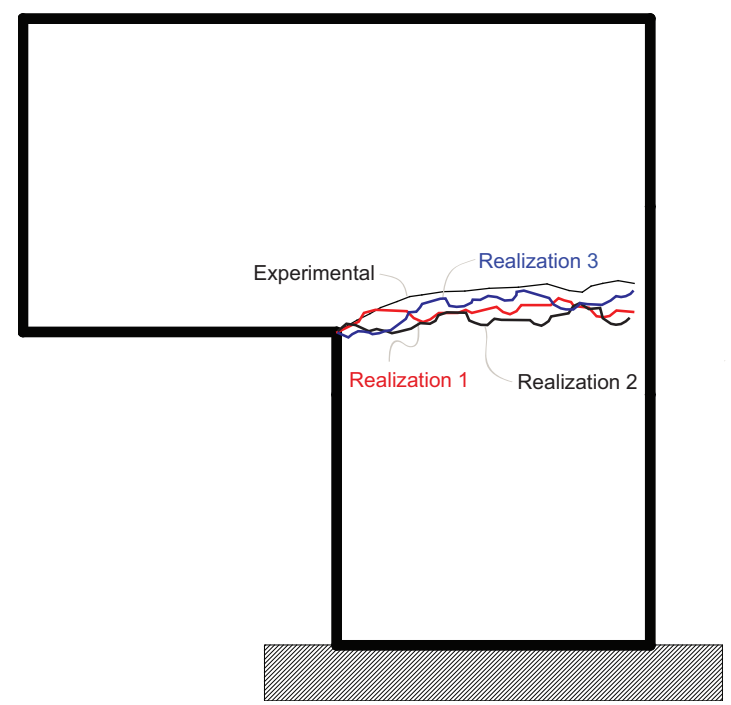

(b) Comparison between the crack pattern numerically and experimentally obtained.

Figure 22. Numerical results computed with the proposed model compared with the experimental results published by Winkler et al. (2001). Coarse Aggregate volume $20 \%$. 


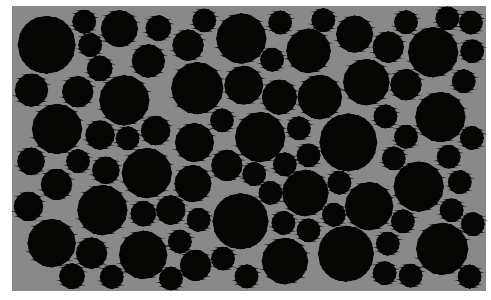

(a) Meso-structure 1.

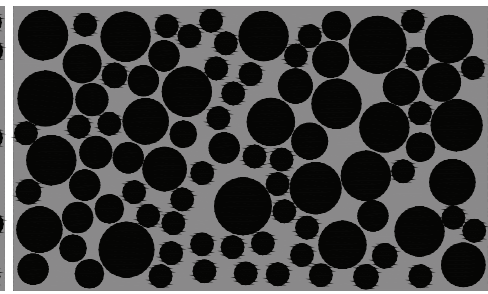

(b) Meso-structure 2.

Figure 23. Meso-structural realizations. Aggregate Volume $60 \%$.
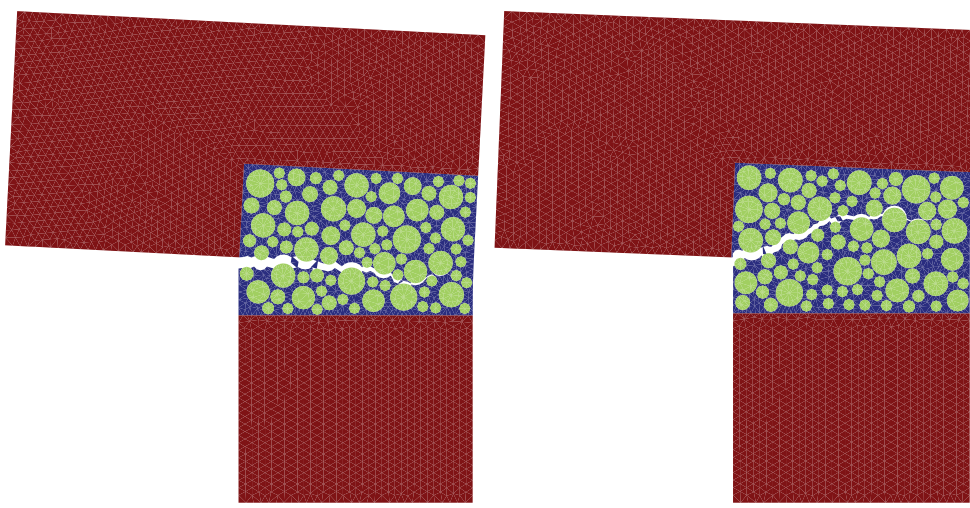

(a) Final configuration for meso- (b) Final configuration for mesomechanical realization 1. Volume aggregate mechanical realization 2. Volume aggregate $60 \%$. Displacements $x 25$.

$60 \%$. Displacements $x 25$.

Figure 24. Simulated structure. Volume aggregate $60 \%$.

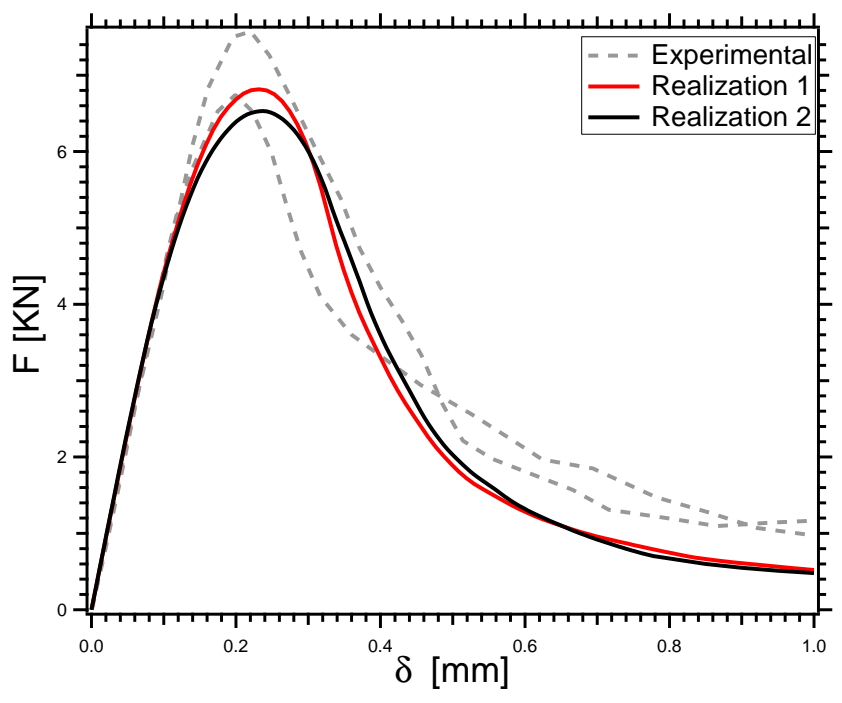

(a) Force versus displacement curves compared with experimental results.

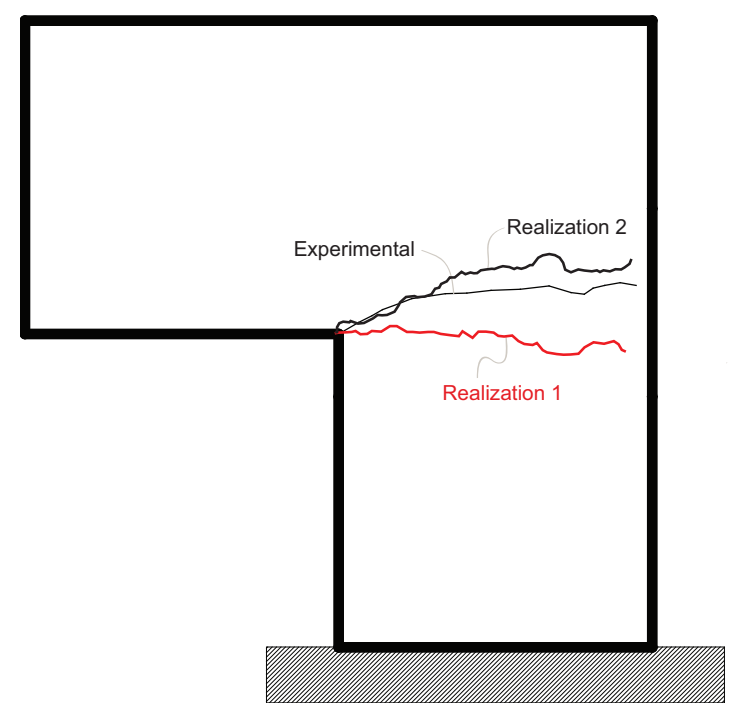

(b) Comparison between the crack pattern numerically and experimentally obtained.

Figure 25. Numerical results computed with the proposed model compared with the experimental results published by Winkler et al. (2001). Coarse Aggregate volume $60 \%$.

on the aggregate content and its distribution in the meso-scale. The effect of particles distribution on crack pattern increases with their content in meso-structure. 


\section{CONCLUSIONS}

A computational fracture method based on an augmented Lagrangian functional to analyze meso-mechanical problems has been presented and discussed. The mathematical equivalence between the solution of the one-field problem and the saddle point of augmented Lagrangian functional via the solution of the double inequality was studied in continuum space. The rigorous description of the model in a variational framework represents an important contribution of the paper. The relation between the Lagrange multipliers, the bulk stress and the cohesive potential was also presented, endowing the mathematical entity with a physical meaning. All the details about the numerical aspects where given for an straightforward computational implementation. The calculation of the supplementary variable $\delta$ and its relation with the TSL with a linear unloading to be implemented in a Newton-Raphson scheme was deeply analysed. An algorithm to adapt classical meshes to the proposed method is also a contribution of the present paper.

A discussion about the selection of the penalty parameter with different interface properties has been presented. It was concluded that a selective parameter $\alpha$ in terms of the material properties is accurate enough, taking care because for $\alpha>10000$ the convergence of the constitutive model for the interface could be not achieved.

Examples with crack propagation in the meso-scale have been presented, showing the ability of the numerical method to reproduce the results obtained with other methodologies and in experimental tests. Experimental result presented in (París et al. 2007) were numerically reproduced and the mechanical response was compared with the obtained using a model based on a discontinuous Galerkin formulation Nguyen (2014). Good results were obtained, even when coarse meshes were used. This particularity is due to the fact that the presented method computes crack tractions directly in the desired point over the fracture domain (using the Lagrange multipliers), avoiding stress recoveries used in discontinuous Galerkin formulations. Afterwards, an expanded example is presented comparing the results with those obtained by Cid Alfaro et al. (2010). Good agreement between both approaches results was obtained even when coarse meshes were used.

Finally, a validation example where the numerical results are compared with those experimentally obtained by Winkler et al. (2001) was presented. The influence of the coarse aggregate content was analysed, generating different models for $20 \%, 50 \%$ and $60 \%$ aggregate volume. The force displacement curves numerically obtained present a good agreement with the experimental results. It was proved that the peak load increases with the decrease of aggregate content. Nonetheless, the influence is low despite the great difference between the contents considered. A different scenario was reached in the post-peak behavior, being almost the same result for all cases. When the fracture pattern was analyzed, it could be seen that in all cases it has a strong dependence with the aggregate distribution. The three hypothesis considered to state this model, seems to be good enough to model fractured concrete at the meso-scale, although they should be proved for different load configurations.

\section{ACKNOWLEDGEMENTS}

The authors wish to thank the financial support of National Agency for Scientific and Technological Promotion, National Scientific and Technological Research Council (CONICET) and National University of Tucumán research Council (CIUNT). The work of S.M. Giusti was partially supported by the European Research Council under the European Unions Seventh Framework Programme (FP/2007-2013) / ERC Grant Agreement n. 320815 (ERC Advanced Grant Project "Advanced tools for computational design of engineering materials" COMP-DES-MAT).

\section{References}

D. Dugdale (1960), Yielding of steel sheets containing slits, Journal of the Mechanics and Physics of Solids 8:100-104.

G. Barenblatt (1963), The mathematical theory of equilibrium cracks in brittle fracture, Advances in Applied Mechanics 7:55-129.

K. Park and G.H. Paulino (2011), Cohesive Zone Models: A Critical Review of Traction-Separation Relationships Across Fracture Surfaces.Applied Mechanics Reviews 64:1-20.

A. Cerrone,P. Wawrzynek,A. Nonn, G.H. Paulino and A. Ingraffea (2014), Implementation and verification of the Park-Paulino-Roesler cohesive zone model in 3D, Engineering Fracture Mechanics 120:26-42.

A. Caggiano and G. Etse (2015), Coupled thermomechanical interface model for concrete failure analysis under high temperature, Comput. Methods Appl. Mech. Engrg. 289:498516.

J.F. Unger, S. Eckardt and C. Konke (2007), Modelling of cohesive crack growth in concrete structures with the extended finite element method, Comput. Methods Appl. Mech. Engrg. 196:4087-4100.

C. Linder, D. Rosato and C. Miehe (2011), New finite elements with embedded strong discontinuities for the modeling of failure in electromechanical coupled solids, Comput. Methods Appl. Mech. Engrg. 200:141-161.

C.V. Verhoosel and R. de Borst (2013), A phase-field model for cohesive fracture, International journal of Numerical Methods in Engineering 96:43-62.

T. Rabczuk and E. Samaniego (2008), Discontinuous modelling of shear bands using adaptive mesh free methods, Comput. Methods Appl. Mech. Engrg. 197:641-658.

M. Fortin and R. Glowinski (1983). Augmented lagrangian methods: application to the numerical solution of boundary-value problems.North-Holland - Studies in mathematics and its applications .

M.F. Wheeler, T. Wick a and W. Wollner (2014), An augmented-Lagrangian method for the phase-field approach for pressurized fractures, Comput. Methods Appl. Mech. Engrg. 271: 6985.

C.J. Corbett and R. Sauer (2015), Three dimensional isogeometrically enriched finite elements for frictional contact and mixed mode debonding, Comput. Methods Appl. Mech. Engrg. 284:781-806.

E. Lorentz (2008), A mixed interface finite element for cohesive zone models, Comput. Methods Appl. Mech. Engrg. 198:302-317. 
V.P. Nguyen (2014), An open source program to generate zero-thickness cohesive interface elements, Advances in Engineering Software, 74:27-39.

F. Brezzi (1974), On the existence, uniqueness and approximation of saddle point problems arising from Lagrangian multipliers.RAIRO Analyse Numerique 8:129-151.

D. Doyen, A. Ern and S.Piperno (2010), A three-field augmented lagrangian formulation of unilateral contact problems with cohesive forces.ESAIM: Mathematical Modelling and Numerical Analysis. 44:323-346.

Zhiming Chen (2001). On the augmented Lagrangian approach to Signorini elastic contact problem.Numer. Math. 88:641-659.

J.L. Lions and E. Magenes (1972). Non-homogeneous Boundary Value Problems and Applications.Springer, Berlin.

V.A. Yastrebov (2013). Numerical Methods in Contact Mechanics.ISTE Ltd and John Wiley and Sons, Inc .

Frank H. Clarke (1978). Non smooth Analysis and Optimization.Proceedings of the International Congress of Mathematicians .

Frank H. Clarke (1990). Optimization and Nonsmooth Analysis. Classics in Applied Mathematics by the Society For Industrial And Applied Mathematics (USA) .

K. Park, G. Paulino and J. Roesler (2009). A unified potential-based cohesive model of mixed-mode fracture.Journal of the Mechanics and Physics of Solids 57:891-908.

J. Mergheim, E. Kuhl and P. Steinmann (2004), A hybrid discontinuous Galerkin-interface method for the computational modelling of failure, Communications in Numerical Methods in Engineering 20:511-519.

V.P. Nguyen (2014), Discontinuous Galerkin/extrinsic cohesive zone modeling: Implementation caveats and applications in computational fracture mechanics, Engineering fracture mechanics, 128:37-68.

R. Radovitzky, A. Seagraves, M. Tupek and L. Noels (2011), A scalable 3D fracture and fragmentation algorithm based on a hybrid discontinuous Galerkin cohesive element method, Comput. Methods Appl. Mech. Engrg, 200:326-344.

S. Sadaba, I. Romero, C. Gonzalez and J. Llorca (2015), A stable X-FEM in cohesive transition from closed to open crack, International Journal for Numerical Methods in Engineering, 101:540-570.

J.Baiges, R. Codina, F. Henke, S. Shahmiri and W.A. Wall (2012), A symmetric method for weakly imposing Dirichlet boundary conditions in embedded finite element meshes, International Journal for Numerical Methods in Engineering, 90:636-658.

M. Prechtel, P. Leiva Ronda, R. Janisch, A. Hartmaier, G. Leugering, P. Steinmann and M. Stingl (2011), Simulation of fracture in heterogeneous elastic materials with cohesive zone models, International Journal of Fracture, 168:15-29.

T. J. Truster and A. Masud (2013), A Discontinuous/continuous Galerkin method for modeling of interphase damage in fibrous composite systems, Computational Mechanics, 52:499514.

S. Liu (1993), Delamination and matrix cracking of cross-ply laminates due to a spherical indenter, Composite Structures, 25:257-265.

T. Elguedj, A. Gravouil and A. Combescure (2007), A mixed augmented Lagrangian-extended finite element method for modelling elasticplastic fatigue crack growth with unilateral contact, International journal of Numerical Methods in Engineering, 71:15691597.

P.M.A. Areias, J.M.A. César de Sá, C.A. Conceicao António, J.A.S.A.O. Carneiro and V.M.P. Teixeira (2004), Strong displacement discontinuities and Lagrange multipliers in the analysis of finite displacement fracture problems, Computational Mechanics, $35: 5471$.

R. L. Taylor, J. C. Simo, O. C. Zienkienwicz and A. C. H. Chan (1986), The patch test. A condition for assessing fem convergence, International Journal for Numerical Method in Engineering, 22:39-62.

V. P. Nguyen and H. Nguyen-Xuan (2013), High-order B-splines based finite elements for delamination analysis of laminated composites, Composite Structures, 102:261-275.

F. París, E. Correa and V. Mantic (2007), Kinking of Transversal Interface Cracks Between Fiber and Matrix, Journal of Applied Mechanics, 74:703-716.

A. Hillerborg, M. Modéer and P. Petersson (1976), Analysis of crack formation and crack growth in concrete by means of fracture mechanics and finite elements, Cement and Concrete Research, 6:773-781.

A. Needleman (1987), A continuum model for void nucleation by inclusion debonding, Journal of Applied Mechanics, 54:525-531.

V. Tvergaard (1987), Effect of fibre debonding in a whisker-reinforced metal, Material Science and Engineering: A, 125:203-213.

H. Inglis, P. Geubelle, K. Matous, H. Tan and Y. Huang (2007), Cohesive modeling of dewetting in particulate composites: micromechanics vs. multiscale finite element analysis, Mechanics of Materials, 41:580-595.

V. Tvergaard and H. Hutchinson (1993), The influence of plasticity on mixed mode interface toughness, Journal of Mechanics and Physics of Solids, 41:1119-1135.

P. Geubelle and J. Baylor (1993), Impact-induced delamination of composites: a 2D simulation, Composites Part B: Engineering, 29:589602.

A. Pantano and R. Averill (2004), A mesh-independent interface technology for simulation of mixed-mode delamination growth, International Journal of Solids and Structures, 14:3809-3831.

A. Turon, C. Dávila, P. Camanho and J. Costa (2007), An engineering solution for mesh size effects in the simulation of delamination using cohesive zone models, Engineering Fracture Mechanics, 70:1665-1682.

L. Wu, D. Tjahjanto, G. Becker, A. Makradi, A. Jrusalem and L. Noels (2013), A micromeso-model of intra-laminar fracture in fiberreinforced composites based on a discontinuous Galerkin/cohesive zone method, Engineering Fracture Mechanics, 104:162 - 183.

M. Cid Alfaro, A. Suiker and R. de Borst (2010), Transverse Failure Behavior of Fiber-epoxy Systems, Journal of Composite Materials, 44:1493-1516.

X. Xu and A. Needleman (1993), Void nucleation by inclusion debonding in a crystal matrix, Modelling and Simulation in Materials Science and Engineering, 1:111-132.

S. Toro, P.J. Sánchez, P.J. Blanco, E.A. de Souza Neto, A.E. Huespe and R.A. Feijóo (2016), Multiscale formulation for material failure accounting for cohesive cracks at the macro and micro scales, International Journal of Plasticity, 76:75-110.

J.F. Unger and S. Eckardt (2011), Multiscale modeling of concrete, Archives of Computational Methods in Engineeringy, 18:75-86.

I. Carol, C.M. López and O.Roa (2001), Micromechanical analysis of quasi-brittle materials using fracture-based interface elements, International Journal of Numerical Methods in Engineering, 52:193-215.

T. Hobbiebrunken, M. Hojo, T. Adachi, C. De Jong and B. Fiedler (2006), Evaluation of interfacial strength in CF/epoxies using FEM and in-situ experiments, Composites: Part A, 37:22482256.

B. Winkler, G. Hofstetter and G. Niederwanger (2001), Experimental verification of a constitutive model for concrete cracking, Proceedings of the Institution of Mechanical Engineers, Part L: Journal of Materials Design and Applications, 215:75-86. 
Xiuli Du, Liu Jin and Guowei Ma(2013), Numerical modeling tensile failure behavior of concrete at mesoscale using extended finite element method, International Journal of Damage Mechanics, 23:872-898.

P. Roelfstra, H. Sadouki and F. Wittmann (1985), Le béton numérique, Materials and Structures, 18:327335.

Z. Bazant, M. Tabbara ,M. Kazemi and G. Pijaudier-Cabot (1990), Random particle model for fracture of aggregate or fiber composites, Engineering Mechanics, ASCE, 116:16861705.

I. Carol, C. López and O. Roa (2001), Micromechanical analysis of quasi-brittle materials using fracture-based interface elements, International Journal of Numerical Methods in Engineering, 52:193215.

C. López, I. Carol, and A. Aguado (2008), Meso-structural study of concrete fracture using interface elements. I: numerical model and tensile behavior, Materials and Structures, 41:583599.

E. A. Rodrigues, O. L. Manzoli and L.A.G. Bitencourt Jr. and T. N. Bittencourt (2016), 2D mesoscale model for concrete based on the use of interface element with a high aspect ratio, International Journal of Solids and Structures, 94:112 - 124.

E. N. Landis and J. E. Bolander (2009), Explicit representation of physical processes in concrete fracture, Journal Of Physics D: Applied Physics, 42. 